\title{
Exact Solution of Kinetic Analysis for Thermally Activated Delayed Fluorescence Materials
}

Youichi Tsuchiya, ${ }^{1 *}$ Stefan Diesing, ${ }^{2,3}$ Fatima Bencheikh,,${ }^{1}$ Yoshimasa Wada, ${ }^{4}$ Paloma Dos Santos, ${ }^{2}$ Hironori Kaji, ${ }^{4}$ Eli

Zysman-Colman, ${ }^{3 *}$ Ifor D. W. Samuel, ${ }^{2 *}$ and Chihaya Adachi ${ }^{1,5,6 *}$

1. Center for Organic Photonics and Electronics Research (OPERA), Kyushu University, 744, Motooka, Nishi-ku, Fukuoka 819-0395, Japan

2. Organic Semiconductor Centre, SUPA School of Physics and Astronomy, University of St Andrews, St Andrews, Fife, UK, KY16 9ST.

3. Organic Semiconductor Centre, EaStCHEM School of Chemistry, University of St Andrews, St Andrews, Fife, UK.

4. Institute for Chemical Research, Kyoto University, Uji, Kyoto, Japan

5. Department of Chemistry and Biochemistry, Kyushu University, Fukuoka, Japan.

6. International Institute for Carbon Neutral Energy Research (WPI-I²CNER), Kyushu University, Fukuoka, Japan.

\begin{abstract}
The photophysical analysis of thermally activated delayed fluorescence (TADF) materials has become instrumental to providing insight into their stability and performance, which is not only relevant for organic lightemitting diodes (OLED), but also for other applications such as sensing, imaging and photocatalysis. Thus, a deeper understanding of the photophysics underpinning the TADF mechanism is required to push materials design further. Previously reported analyses in the literature of the kinetics of the various processes occurring in a TADF material rely on several a priori assumptions to estimate the rate constants for forward and reverse intersystem crossing (ISC and RISC, respectively). In this report, we demonstrate a method to determine these rate constants using a three-state model together with a steady-state approximation and, importantly, no additional assumptions. Further, we derive the exact rate equations, greatly facilitating a comparison of the TADF properties of structurally diverse emitters and providing a comprehensive understanding of the photophysics of these systems.
\end{abstract}

\section{Introduction}

In recent years, organic thermally activated delayed fluorescence (TADF) materials have attracted significant attention within the organic semiconductor community as TADF provides a route for $100 \%$ internal quantum efficiency in organic light-emitting diodes (OLEDs), without the need to use precious noble metal complexes. ${ }^{1-4}$ Distinct from phosphors that rely on large spin-orbit coupling to drive the conversion of singlet excitons to triplets and then phosphorescence of the latter, organic TADF materials convert triplet excitons to singlet excitons by taking advantage of the small energy gap $\left(\Delta \mathrm{EST}_{\mathrm{ST}}\right)$, typically taken as less than $200 \mathrm{meV}$, between the lowest singlet $\left(\mathrm{S}_{1}\right)$ and triplet $\left(\mathrm{T}_{1}\right)$ excited states. As $\mathrm{T}_{1}$ excitons are efficiently upconverted into an $\mathrm{S}_{1}$ level through a reverse intersystem crossing (RISC) route, OLEDs can harvest the $75 \%$ of electrically generated excitons that are triplets for electroluminescence (EL) as 
delayed fluorescence from the singlet excited state. The most common molecular design that shows small $\Delta$ Est is based on a donor-acceptor architecture wherein there is poor electronic communication between the two moieties, resulting in a small exchange integral and a correspondingly small $\triangle$ EsT. TADF materials typically exhibit dual fluorescence consisting of a prompt nanosecond fluorescence originating from the radiative decay of directly formed singlet excitons and a microsecond to millisecond delayed fluorescence that originates from the multiple ISC and RISC cycles preceding emission from the singlet excited state. The performance of the OLED, and in particular the efficiency roll-off and the device stability, are intimately linked to the population of the long-lived triplet excitons. As RISC is the slowest process typically observed in OLEDs using organic TADF emitters, the optimization of the device performance is intimately linked to increasing the rate constant associated with RISC, $k$ RISC. Thus, it is essential to have an accurate measure of this key rate constant.

Many researchers have tried to understand the photophysics of TADF materials by curve fitting the time-resolved photoluminescence (PL) decays using a conventional rate equation strategy that accounts for each decay process. While this approach is useful, most previous analyses require several a priori assumptions, leading to rather large deviations from experiment or, in some cases, completely incorrect conclusions. For example, in 1983, McMillin and co-workers explained the TADF behavior of a $\mathrm{Cu}(\mathrm{I})$ complex using a Boltzmann statistical analysis of the population equilibrium of excitons in the $S_{1}$ and $T_{1}$ states, this analysis based on several assumptions. These included that the intersystem crossing (ISC) efficiency $\left(\Phi_{I S C}\right)$ is almost unity and the rate constant of nonradiative decay from the singlet excited state $\left(k_{n r}^{S}\right)$ is $0 .{ }^{5}$ In a very early study in 2012, our group applied this method to organic TADF materials having nearly $100 \%$ PL quantum yield (PLQY). ${ }^{6}$ Here, $k_{R I S C}$ was given by

$$
k_{R I S C}=\frac{1}{3} k_{r}^{S} \exp \left(\frac{-\Delta E_{S T}}{R T}\right)
$$

where $R$ and $T$ are the ideal gas constant and the temperature, respectively. However, the materials that can be analyzed using this method are quite limited because of the demanding assumptions this model makes. Subsequently, in our first kinetics analysis model in 2012, $k_{R I S C}$ was obtained but with a less stringent set of assumptions that the radiative and nonradiative decay rate constant from a singlet excited state $\left(k_{r}^{S}\right)$ and $k_{n r}^{S}$, respectively, and the ISC rate constant $\left(k_{I S C}\right)$ are all significantly larger than both the rate constants of nonradiative decay from a triplet excited state $\left(k_{n r}^{T}\right)$ and $k_{R I S C}$; further, it was assumed that radiative decay from a triplet excited state $\left(k_{r}^{T}\right)$ does not occur. ${ }^{7}$ In our second, revised, kinetics analysis study, we further simplified the model by imposing the additional assumption that $k_{n r}^{S}=0,{ }^{8}$ which implies that $k_{r}^{S}$ and $k_{I S C}$ are significantly larger than $k_{n r}^{S}$ and $k_{R I S C}$. Within this framework, $k_{I S C}$ and $k_{R I S C}$ can be formulated as:

$$
\begin{aligned}
& k_{R I S C}=\frac{k_{p} k_{d}}{k_{I S C}} \cdot \frac{\Phi_{D E}}{\Phi_{P F}}, \\
& k_{I S C}=k_{p}\left(1-\Phi_{P F}\right),
\end{aligned}
$$

where $\Phi_{P F}, \Phi_{D E}, k_{p}$ and $k_{d}$ are the experimentally obtained PL efficiencies and decay rates for the prompt and delayed emissions. In 2016, Wu, Wong and co-workers arrived at the same equations as those of Eqs. 1.2 and 1.3, but 
by imposing fewer assumptions in their model. ${ }^{9}$ In the same paper, they also obtained the following equation with the assumption of $k_{r}^{T}=k_{n r}^{T}=0$ as:

$$
k_{R I S C}=\frac{k_{p} k_{d}}{k_{r}^{S}} \Phi_{P L Q Y}
$$

Dias, Penfold and Monkman more recently proposed a model that applies for TADF emitters that show a large fraction of delayed emission, i.e., $\Phi_{D E} / \Phi_{P F}>4$, and with the assumptions that $k_{r}^{T}=k_{n r}^{T}=0 .{ }^{10}$

$$
k_{R I S C}=k_{d} \frac{\Phi_{P F}+\Phi_{D E}}{\Phi_{P F}}
$$

Kaji and co-workers removed the constraint that $\Phi_{D E} / \Phi_{P F}>4$ and showed that $k_{R I S C}$ could be estimated with only the assumptions that $k_{r}^{T}=k_{n r}^{T}=0 .{ }^{11}$

$$
k_{R I S C}=\frac{k_{p}+k_{d}}{2}-\sqrt{\left(\frac{k_{p}+k_{d}}{2}\right)^{2}-k_{p} k_{d}\left(1+\frac{\Phi_{D E}}{\Phi_{P F}}\right)} .
$$

Taking each a different approach, Hasse, Brütting, Monkman and co-workers demonstrated the direct fitting of the time-resolved PL decays using the rate constants as fitting parameters, ${ }^{12}$ while Nguyen et al. provided an analysis model derived from experiments employing an exciton quencher. ${ }^{13}$ Goodson and co-workers extracted the triplet decay rate using transient absorption (TA) measurement as a proxy for the RISC rate constant, but they did not appear to consider cycling between the singlets and triplets. ${ }^{14}$ The constraints and assumptions for these RISC rate constant estimations are summarized in Table S1. Since there are now a large number of different models used to estimate $k_{\text {RISC }}$, each with their own set of assumptions, it has become impossible to accurately compare the estimated rate constants across these studies, complicating any meta-analysis. To reduce the number of assumptions and the differences in estimated $k_{R I S C}$, $\Phi_{I S C}$ must be measured experimentally.

Because $\Phi_{I S C}$ and its related rate constant $k_{I S C}$ are two of the essential parameters of photochemical processes that implicate triplet states, dating back to before 1970, several groups have focused on an estimation method of accurate $\Phi_{I S C}$. Scott and Maltenieks proposed a method to estimate $k_{I S C}$ using triplet absorption under steady-state conditions. ${ }^{16}$ To apply this method to TADF materials, their equation can be rewritten as

$$
\Phi_{I S C}=\frac{\rho(\lambda)_{S S} k_{d}}{\varepsilon_{T}(\lambda) I_{a} l}
$$

where $\rho(\lambda)_{S S}$ is the optical density change due to the triplet-triplet absorption from a $\mathrm{T}_{1}$ state to a higher-lying triplet $\left(\mathrm{T}_{\mathrm{n}}\right)$ state at the steady-state condition, $\varepsilon_{T}(\lambda)$ is the triplet-triplet extinction coefficient, $I_{a}$ is the rate of absorption of exciting light, and $l$ is the optical path length. It should be noted that $\varepsilon_{T}(\lambda)$ can be estimated experimentally. ${ }^{17}$ In another study, Berberan-Santos et al. provided an estimation of $\Phi_{I S C}$ by fitting the intensity ratio of the prompt and delayed emission $\left(I_{P F} / I_{D F}\right)$, corresponding to $\Phi_{P L}^{p} / \Phi_{P L}^{d}$, vs $1 / T$ by using Eq. $1.8 .^{18}$

$$
\ln \left[\frac{I_{P F}}{I_{D F}}-\left(\frac{1}{\Phi_{I S C}}-1\right)\right]=\frac{\Delta E_{S T}}{R} \cdot \frac{1}{T}+\ln \left(\frac{k_{r}^{T}+k_{n r}^{T}}{\bar{k}_{R I S C}}\right),
$$

where $\bar{k}_{R I S C}$ is the average rate constant for RISC. The shape of the plot is very sensitive to $\Phi_{I S C}$, and is normally not linear. Continuous variation of $\Phi_{I S C}$ within the search for maximum linearity yields its best value. It should be noted 
that this method assumes that $\left(k_{r}^{T}+k_{n r}^{T}\right)$ and $\Phi_{I S C}$ are each essentially temperature independent. They also provided another analysis method for $\Phi_{I S C}$, shown in Eq. 1.9, which combines a steady-state condition and a time-resolved analysis. ${ }^{19}$

$$
\tau_{D F}=\tau_{P h o s}^{0}-\left(\frac{1}{\Phi_{I S C}}-1\right) \tau_{P h o s}^{0} \frac{I_{D F}}{I_{P F}}
$$

where $\tau_{D F}$ is the delayed fluorescence lifetime $\left(1 / k_{d}\right)$ and $\tau_{P h o s}^{0}$ is the low-temperature phosphorescence lifetime without any contribution to the emission associated with TADF, which means $1 /\left(k_{r}^{T}+k_{n r}^{T}\right)$. This method also assumes that $\left(k_{r}^{T}+k_{n r}^{T}\right)$ is temperature independent. Our group demonstrated an estimation of $\Phi_{I S C}$ for thin film samples by combining pulse-excited electroluminescence (EL) and PL measurements. ${ }^{7}$ Here, $\Phi_{I S C}$ can be obtained as

$$
\Phi_{I S C}=\frac{3 N}{M-N}
$$

where $M$ and $N$ are the quantum yield ratio of delayed fluorescence and prompt fluorescence for EL and PL, respectively; $\Phi_{E L}^{d} / \Phi_{E L}^{p}=M, \Phi_{P L}^{d} / \Phi_{P L}^{p}=N$. This method is very effective since it requires only the ratio of initial exciton distribution between $S_{1}$ and $T_{1}$ states, although this model is only relevant for thin films where OLED driving is applicable. Very recently, Naito et al. reconsidered the estimation method to obtain $k_{I S C}$ from PL decay measurements under the assumption of $k_{r}^{S}+k_{I S C} \gg k_{R I S C} \gg k_{r}^{T}+k_{n r}^{T}$, (Eq. 1.11). ${ }^{20}$ This is a reasonable assumption for efficient organic TADF emitters.

$$
\Phi_{I S C}=\frac{\Phi_{D F}+\Phi_{P h o s}}{\Phi_{r}^{S}+\Phi_{D F}+\Phi_{P h o s}}=\frac{\Phi_{D E}}{\Phi_{P L Q Y}} .
$$

This equation for $\Phi_{I S C}$ converges to our three-state analysis (see Eq. S6.4) when $\Phi_{n r}^{T}=0$ and $\Phi_{r}^{T}=0$ are employed; however, we note that Eq. 1.11 is derived without these assumptions. This method should only be applied at around room temperature for organic TADF materials where triplet excitons mainly decay by a RISC path. Naito et $a l$. also provided an equation for $\Phi_{I S C}$ determined at low temperature where the TADF is completely suppressed. For the temperature region where contributions from nonradiative decay paths cannot be ignored, an alternative approach using the temperature dependence of the prompt fluorescence rate is also provided.

Thus, if thin film samples are available, our model using Eq. 1.10 is the most promising and provides the most accurate determination of $\Phi_{I S C}$. Without considering the complexity of the experimental setup and the associated specialized instrumentation, the method proposed by Scott and Maltenieks should be employed for solution-state samples. Both methods provided by Berberan-Santos et al. assume that $\left(k_{r}^{T}+k_{n r}^{T}\right)$ is temperature independent, which is a limitation of their methods as $k_{n r}^{T}$ usually possesses a temperature dependence that is explained in terms of the thermal quench model. ${ }^{21,22}$ The method of Naito et al. should be useful as it does not assume temperature independence for $\left(k_{r}^{T}+k_{n r}^{T}\right)$; however, it cannot be applied to inefficient TADF materials.

As explained above, most of the previously reported rate equations used for TADF compounds are based on models derived from highly emissive materials and assume $\Phi_{n r}^{S}=0$ or $\Phi_{n r}^{T}=0$, in order to obtain an estimation of $\Phi_{I S C}$. Therefore, most of these previously reported rate equations are not appropriate for the analysis of low efficiency TADF materials. However, we often find reports that uncritically employ these models and equations to extract rate 
constants for low efficiency materials. In addition, assumptions such as $\Phi_{n r}^{S}=0$ or $\Phi_{n r}^{T}=0$ raise the question about "which parameter should be set to zero". Here, we introduce two kinetics analysis methods for TADF materials based on a three-state system of $S_{0}, S_{1}$, and $T_{1}$. In our first method where we invoke a steady-state approximation, no other assumptions are required in order to derive the set of rate constants related to the emission of TADF materials. This method not only can help in our understanding of TADF processes, but it can demonstrate the connections between several previously reported methods; however, this method still has the weakness in terms of providing an accurate estimation of $\Phi_{I S C}$. To resolve this outstanding issue, we then present a derived exact solution to provide precise rate constants in the kinetics analysis of TADF materials.

\section{Exact equation for emission decay curve for a three-state system}

We first present an exact equation to model the emission decay that occurs from a three-state system comprising $\mathrm{S}_{1}$ and $\mathrm{T}_{1}$ coupled excited states and the $\mathrm{S}_{0}$ ground state (Figure 1 ). The combined decay rates from both excited states in the absence of exciton-formation processes can be formulated as

$$
\begin{aligned}
& \frac{d\left[\mathrm{~S}_{1}\right]}{d t}=-\left(k_{r}^{S}+k_{n r}^{S}+k_{I S C}\right)\left[\mathrm{S}_{1}\right]+k_{R I S C}\left[\mathrm{~T}_{1}\right], \\
& \frac{d\left[\mathrm{~T}_{1}\right]}{d t}=-\left(k_{r}^{T}+k_{n r}^{T}+k_{R I S C}\right)\left[\mathrm{T}_{1}\right]+k_{I S C}\left[\mathrm{~S}_{1}\right],
\end{aligned}
$$

where $\left[\mathrm{S}_{1}\right]$ and $\left[\mathrm{T}_{1}\right]$ are the populations of $\mathrm{S}_{1}$ and $\mathrm{T}_{1}$ excitons. The differential equations, Eqs. 2.1 and 2.2, are a system of ordinary differential equations of the general formula of $d \overrightarrow{\mathrm{Y}}(t) / d t=A \overrightarrow{\mathrm{Y}}(t)$, and can be written as,

$$
\frac{d}{d t}\left(\begin{array}{l}
{\left[\mathrm{S}_{1}\right]} \\
{\left[\mathrm{T}_{1}\right]}
\end{array}\right)=\left(\begin{array}{cc}
-k^{S} & k_{R I S C} \\
k_{I S C} & -k^{T}
\end{array}\right)\left(\begin{array}{l}
{\left[\mathrm{S}_{1}\right]} \\
{\left[\mathrm{T}_{1}\right]}
\end{array}\right)
$$

where $k^{S}=k_{r}^{S}+k_{n r}^{S}+k_{I S C}$ and $k^{T}=k_{r}^{T}+k_{n r}^{T}+k_{R I S C}$, respectively. The general solution to this system is given by,

$$
\vec{Y}(t)=\sum_{i=1}^{n} c_{i} \overrightarrow{v_{l}} \exp \left(\lambda_{i} t\right)
$$

where $\overrightarrow{v_{l}}$ is the eigenvector with corresponding eigenvalue $\lambda_{i}$ of the matrix $A$, and $c_{i}$ is a constant depending on the initial conditions. For the matrix $A$ in Eq. 2.3, the eigenvalues can be calculated as,

$$
\operatorname{det}(A-\lambda E)=\operatorname{det}\left[\left(\begin{array}{cc}
-k^{S}-\lambda & k_{R I S C} \\
k_{I S C} & -k^{T}-\lambda
\end{array}\right)\right]=\left(k^{S}+\lambda\right)\left(k^{T}+\lambda\right)-k_{I S C} k_{R I S C}=0,
$$

where $E$ is the identity matrix. Eq. 2.5 can be rewritten as,

$$
\lambda^{2}+\left(k^{S}+k^{T}\right) \lambda+k^{S} k^{T}-k_{I S C} k_{R I S C}=0 .
$$

This quadratic equation provides $\lambda$ as,

$$
\lambda_{1,2}=-\frac{k^{S}+k^{T}}{2} \pm \sqrt{\frac{\left(k^{S}+k^{T}\right)^{2}}{4}-k^{S} k^{T}+k_{I S C} k_{R I S C}}=-\frac{1}{2}\left(k^{S}+k^{T} \mp \sqrt{\left(k^{S}-k^{T}\right)^{2}+4 k_{I S C} k_{R I S C}}\right) .
$$


Here, it is worth pointing out that $\lambda_{1}<\lambda_{2}<0$ and, therefore, the exact prompt and delayed emission decays for the photoluminescence $\left(k_{p}, k_{d}\right)$ are written as $k_{p}=-\lambda_{1}$ and $k_{d}=-\lambda_{2}$, respectively. Several rate equations introduced in the previous section have been derived from Eq. 2.7 but employ a number of different assumptions. Here, we note that Eq. 2.7 provides a relationship among $k^{S}, k^{T}, k_{p}$, and $k_{d}$ as,

$$
k^{S}+k^{T}=k_{p}+k_{d}
$$

Considering the clear relationships of $k^{\mathrm{S}}>k_{d}$ and $k_{p}>k^{T}$, Eq. 2.5 implies the relationships of $k_{p}>k^{S}$ and $k^{T}>$ $k_{d}$ (these provided relationships are explained again in section 5). If these relationships are not operative, then there should be no observed delayed emission. The corresponding eigenvectors are given by evaluating $\lambda_{i} \overrightarrow{v_{l}}=A \overrightarrow{v_{l}}$.

$$
\lambda_{i}\left(\begin{array}{l}
x_{1} \\
x_{2}
\end{array}\right)=\left(\begin{array}{cc}
-k^{S} & k_{R I S C} \\
k_{I S C} & -k^{T}
\end{array}\right)\left(\begin{array}{l}
x_{1} \\
x_{2}
\end{array}\right) .
$$

Eq. 2.9 leads to the relationship of Eq. 2.10.

$$
\left\{\begin{array} { l } 
{ x _ { 1 } = \frac { k _ { R I S C } } { k ^ { S } + \lambda _ { i } } x _ { 2 } } \\
{ x _ { 2 } = \frac { k _ { I S C } } { k ^ { T } + \lambda _ { i } } x _ { 1 } }
\end{array} \Leftrightarrow \left\{\begin{array} { l } 
{ x _ { 1 } = \frac { k _ { R I S C } } { k ^ { S } + \lambda _ { i } } x _ { 2 } } \\
{ x _ { 2 } = x _ { 2 } , \quad x _ { 2 } \in \mathbb { R } }
\end{array} \Leftrightarrow \left\{\begin{array}{l}
x_{1}=x_{1}, \quad x_{1} \in \mathbb{R} \\
x_{2}=\frac{k_{I S C}}{k^{T}+\lambda_{i}} x_{1}
\end{array} .\right.\right.\right.
$$

Using Eq 2.5, this set of equations can be easily reduced to a linear relationship between the dimensions of the eigenvector, allowing for the other dimension to be fixed to a freely chosen value. When $x_{2}=1$ is chosen, both eigenvectors, $\overrightarrow{v_{1}}$ and $\overrightarrow{v_{2}}$ are given by

$$
\overrightarrow{v_{1}}=\left(\begin{array}{c}
\frac{k_{R I S C}}{k^{S}+\lambda_{1}} \\
1
\end{array}\right), \quad \overrightarrow{v_{2}}=\left(\begin{array}{c}
\frac{k_{R I S C}}{k^{S}+\lambda_{2}} \\
1
\end{array}\right) .
$$

Inserting Eq. 2.11 in Eq. 2.4 yields the following equations for $\left[\mathrm{S}_{1}\right]$ and $\left[\mathrm{T}_{1}\right]$ as a function of time.

$$
\begin{aligned}
& \left(\begin{array}{l}
{\left[\mathrm{S}_{1}\right]} \\
{\left[\mathrm{T}_{1}\right]}
\end{array}\right)=c_{1}\left(\begin{array}{c}
\frac{k_{R I S C}}{k^{S}+\lambda_{1}} \\
1
\end{array}\right) \exp \left(\lambda_{1} t\right)+c_{2}\left(\frac{k_{R I S C}}{k^{S}+\lambda_{2}}\right) \exp \left(\lambda_{2} t\right), \\
& \left\{\begin{array}{c}
{\left[\mathrm{S}_{1}\right]=c_{1} \frac{k_{R I S C}}{k^{S}-k_{p}} \exp \left(-k_{p} t\right)+c_{2} \frac{k_{R I S C}}{k^{S}-k_{d}} \exp \left(-k_{d} t\right)} \\
{\left[\mathrm{T}_{1}\right]=c_{1} \exp \left(-k_{p} t\right)+c_{2} \exp \left(-k_{d} t\right)}
\end{array} .\right.
\end{aligned}
$$

The pre-exponential factors depend on the initial conditions. Using $\left[\mathrm{S}_{1}\right]=\left[\mathrm{S}_{1}\right]_{t=0}$ and $\left[\mathrm{T}_{1}\right]=\left[\mathrm{T}_{1}\right]_{t=0}=0$ at $t=$ 0 , they are expressed as follows.

$$
\left(\begin{array}{c}
{\left[\mathrm{S}_{1}\right]_{t=0}} \\
0
\end{array}\right)=c_{1}\left(\begin{array}{c}
\frac{k_{R I S C}}{k^{S}-k_{p}} \\
1
\end{array}\right)+c_{2}\left(\frac{k_{R I S C}}{k^{S}-k_{d}}\right)
$$

Eq. 2.14 expresses the relationship of Eq. 2.15.

$$
\left\{\begin{array}{c}
{\left[\mathrm{S}_{1}\right]_{t=0}=c_{1} \frac{k_{R I S C}}{k^{S}-k_{p}}+c_{2} \frac{k_{R I S C}}{k^{S}-k_{d}} .} \\
c_{1}=-c_{2}
\end{array}\right.
$$

Here, $\left[\mathrm{S}_{1}\right]_{t=0}$ and $c_{2}$ are given as Eqs. 2.16 and 2.17.

$$
\left[\mathrm{S}_{1}\right]_{t=0}=c_{2} \frac{k_{R I S C}\left(k_{p}-k_{d}\right)}{\left(k_{p}-k^{S}\right)\left(k^{S}-k_{d}\right)},
$$




$$
c_{2}=\left[\mathrm{S}_{1}\right]_{t=0} \frac{k_{I S C}}{k_{p}-k_{d}} .
$$

From Eqs. 2.13, 2.16, and 2.17, the exact $\left[\mathrm{S}_{1}\right]$ and $\left[\mathrm{T}_{1}\right]$ can be written as following equations.

$$
\begin{gathered}
{\left[\mathrm{S}_{1}\right]=\frac{\left[\mathrm{S}_{1}\right]_{t=0}}{k_{p}-k_{d}}\left[\left(k^{S}-k_{d}\right) \exp \left(-k_{p} t\right)+\left(k_{p}-k^{S}\right) \exp \left(-k_{d} t\right)\right] .} \\
{\left[\mathrm{T}_{1}\right]=\frac{\left[\mathrm{S}_{1}\right]_{t=0} k_{I S C}}{k_{p}-k_{d}}\left[-\exp \left(-k_{p} t\right)+\exp \left(-k_{d} t\right)\right] .}
\end{gathered}
$$

Here, it is evident that Eq. 2.18 provides a bi-exponential decay of the $\mathrm{S}_{1}$ population, and Eq. 2.19 provides a convex curve behavior of the evolution of the $\mathrm{T}_{1}$ population. Hasse et al. reported that the emission decay of TADF materials corresponds to the $\mathrm{S}_{1}$ population decay when assuming $k_{r}^{T}=k_{n r}^{T}=0 .{ }^{12}$ They also verified that the depletion of the $\mathrm{T}_{1}$ population was accurately determined by the TA decay. When now including the depletion from the $\mathrm{T}_{1}$ state term described in Eq. 2.19, the total emission decay now includes the contributions from both $\mathrm{S}_{1}$ and $\mathrm{T}_{1}$ populations. Because the total emission from $S_{1}$ is the sum of the overall emission efficiencies from $S_{1}$ excitons, which are generated by direct photoexcitation and indirectly from the $\mathrm{T}_{1}$ population, i.e., sum of prompt and delayed fluorescence $\left(\Phi_{P F}+\Phi_{D F}\right)$, the contribution arising from generated triplet excitons must also be accounted for within the emission efficiency from

$\mathrm{T}_{1}$, phosphorescence $\left(\Phi_{\text {Phos }}\right)$. The $\mathrm{S}_{1}$ and $\mathrm{T}_{1}$ populations described by Eqs. 2.18 and 2.19 do not consider the luminescence. Therefore, the exact emission decay can be modelled as a bi-exponential decay as described in Eq. 2.20 using the radiative decay ratio for each exciton (Figure 2).

$$
\begin{aligned}
I(t) & =\Phi_{r}^{S}\left[\mathrm{~S}_{1}\right]+\Phi_{r}^{T}\left[\mathrm{~T}_{1}\right] \\
& =\frac{\left[S_{1}\right]_{t=0}}{k_{p}-k_{d}}\left\{\left[\left(k^{S}-k_{d}\right) \Phi_{r}^{S}-k_{I S C} \Phi_{r}^{T}\right] \exp \left(-k_{p} t\right)+\left[\left(k_{p}-k^{S}\right) \Phi_{r}^{S}+k_{I S C} \Phi_{r}^{T}\right] \exp \left(-k_{d} t\right)\right\} .
\end{aligned}
$$

where $I(t)$ is the time-dependent emission intensity, $\Phi_{r}^{S}$ and $\Phi_{r}^{T}$ are the quantum efficiencies of the radiative decay for $\mathrm{S}_{1}$ and $\mathrm{T}_{1}$ as written by and $k_{r}^{S} / k^{S}$ and $k_{r}^{T} / k^{T}$, respectively. By using this equation that describes explicitly the emission decay, we can deeply understand the kinetics of TADF based on a three-state system.

\section{Derivation of rate equations using steady-state approximation}

In the previous section, we derived the rate equations for the emission decay of TADF materials based on a threestate system. In this section, we derive the rate equations by using the assumption of $k_{r}^{S}+k_{n r}^{S}+k_{I S C} \gg k_{R I S C}$. This assumption implies that at least one of $k_{r}^{S}, k_{n r}^{S}$, or $k_{I S C}$ is much larger than $k_{R I S C}$. Since the $\mathrm{T}_{1}$ level is lying below the $\mathrm{S}_{1}$ level, $k_{I S C} \gg k_{R I S C}$ should always be valid and no further assumptions are employed.

Before deriving the rate equations, we define $\Phi_{P F}$ and $\Phi_{D E}$ in terms of $A_{p}, A_{d}, k_{p}$ and $k_{d}$ which are parameters that describe a bi-exponential decay curve; these parameters are the pre-exponential factors $(A)$ and decay rate constants $(k)$ for the prompt $(p)$ and delayed $(d)$ components, respectively. There are several reported methods to obtain these values experimentally. ${ }^{23}$ For instance, these values can be obtained from the integration of the emission decay corresponding to the prompt and delayed emission, respectively. ${ }^{24}$ Also, there is the rough method of using the 
experimentally determined PLQY under aerated conditions as a surrogate for $\Phi_{P F}$; however, the triplet state may not be completely quenched by $\mathrm{O}_{2}$ under aerated conditions, and it is not the case that oxygen is benign to reaction with the $S_{1}$ state of TADF materials as its presence has been shown to increase the nonradiative decay path from the $S_{1}$ state. ${ }^{15}$ The most commonly used approach to determine these values would be using Eqs. S3.1 and S3.2 where $A_{p}, A_{d}$, $k_{p}$ and $k_{d}$ can be obtained from bi-exponential curve fitting. However, the two exponential curves in Eq. 2.20 do not directly correspond to the exact "prompt emission" and "delayed emission", respectively. To estimate each efficiency, therefore, it is necessary to rewrite Eq. 2.20 as,

$$
I(t)=\left(A_{p}+A_{d}\right) \exp \left(-k_{p} t\right)+A_{d}\left[-\exp \left(-k_{p} t\right)+\exp \left(-k_{d} t\right)\right]
$$

In this form, the first and second terms exactly correspond to the prompt and delayed emission, respectively (Figure 3 ). The quantum efficiency of the prompt $\left(\Phi_{P F}\right)$ and delayed emission $\left(\Phi_{D E}\right)$ are therefore given by,

$$
\begin{gathered}
\Phi_{P F}=\frac{\frac{A_{p}+A_{d}}{k_{p}}}{\frac{A_{p}+A_{d}}{k_{p}}+\frac{A_{d}}{k_{d}}-\frac{A_{p}}{k_{p}}} \Phi_{P L Q Y}=\frac{\left(A_{p}+A_{d}\right) k_{d}}{A_{p} k_{d}+A_{d} k_{p}} \Phi_{P L Q Y}, \\
\Phi_{D E}=\frac{\frac{A_{d}}{k_{d}}-\frac{A_{p}}{k_{p}}}{\frac{A_{p}+A_{d}}{k_{p}}+\frac{A_{d}}{k_{d}}-\frac{A_{p}}{k_{p}}} \Phi_{P L Q Y}=\frac{A_{d}\left(k_{p}-k_{d}\right)}{A_{p} k_{d}+A_{d} k_{p}} \Phi_{P L Q Y},
\end{gathered}
$$

Under the assumption of $k_{r}^{S}+k_{n r}^{S}+k_{I S C} \gg k_{R I S C}$ and with the restriction condition of $\left[\mathrm{S}_{1}\right] \gg\left[\mathrm{T}_{1}\right](t \ll$ $1 / k_{p}$ ), the emission decay agrees with the prompt decay in this region and $\left[\mathrm{T}_{1}\right]$ can be approximated to be 0. Eq. 2.1 can therefore be rewritten as Eq. 3.4. Hence, the singlet decay rate $\left(k^{S}\right)$ can be approximated to the prompt decay rate $\left(k_{p}\right)$, and the evolution of the $\mathrm{S}_{1}$ population as a function of time at short time can be written as Eq. 3.5.

$$
\begin{gathered}
\frac{d\left[\mathrm{~S}_{1}\right]}{\left[\mathrm{S}_{1}\right]} \approx-\left(k_{r}^{S}+k_{n r}^{S}+k_{I S C}\right) d t . \\
{\left[\mathrm{S}_{1}\right] \approx A_{S} \exp \left[-\left(k_{r}^{S}+k_{n r}^{S}+k_{I S C}\right) t\right]=A_{S} \exp \left(-k^{S} t\right) \approx A_{S} \exp \left(-k_{p} t\right),}
\end{gathered}
$$

where $A_{S}$ is a pre-exponential factor.

Next, we focus on the exponential decay of $\left[\mathrm{T}_{1}\right]$ to derive $k_{\mathrm{ISC}}$. Eq. 2.2 can be rewritten as Eq. 3.6.

$$
\frac{d\left[\mathrm{~T}_{1}\right]}{\left[\mathrm{T}_{1}\right]} \approx-\left(k_{r}^{T}+k_{n r}^{T}+k_{R I S C}-k_{I S C} \frac{\left[\mathrm{S}_{1}\right]}{\left[\mathrm{T}_{1}\right]}\right) d t .
$$

Here, since $\left[\mathrm{S}_{1}\right]$ and $\left[\mathrm{T}_{1}\right]$ are time-dependent terms; this equation cannot be integrated. In the delayed decay region, however, we note that the decay rate is not exactly the same as the intrinsic triplet decay rate, as delayed emission decay also contains a term relating to the singlet population given that there is an $\exp \left(-k_{d} t\right)$ term found in the corresponding equations of Eqs. 2.18-2.20. Therefore, the ratio of singlet and triplet population, $\left[\mathrm{S}_{1}\right] /\left[\mathrm{T}_{1}\right]$, is not a time-dependent value but an exactly fixed value in the delayed decay region $\left(t \gg 1 / k_{p}\right)$. Further, the delayed component that originates from an $\mathrm{S}_{1}$ population decay is extremely small compared with the $\mathrm{T}_{1}$ population decay, $\left[\mathrm{T}_{1}\right] \gg\left[\mathrm{S}_{1}\right]$, because $k_{I S C} \gg$ $k_{\text {RISC }}$ (Figure $2 \mathrm{~b}$ ). Therefore, the temporal differentiation of $\left[\mathrm{S}_{1}\right]$ can be approximated to be 0 , i.e., $d\left[\mathrm{~S}_{1}\right] / d t \approx 0$, in this time region. In other word, the population of the intermediate state $S_{1}$ resulting from upconversion of $T_{1}$ excitons, which then decay to $\mathrm{S}_{0}$ can be considered using the steady-state approximation. We thus obtain the ratio of $\left[\mathrm{S}_{1}\right] /\left[\mathrm{T}_{1}\right]$ 
in Eq. 3.6 by using the steady-state approximation (SSA) in this time region. By the SSA, the ratio of $\left[\mathrm{S}_{1}\right] /\left[\mathrm{T}_{1}\right]$ can be provided as Eq. 3.7. Also, the SSA provides a description of the time dependence for $\left[\mathrm{T}_{1}\right]$ as Eq. 3.8.

$$
\begin{gathered}
\frac{\left[\mathrm{S}_{1}\right]}{\left[\mathrm{T}_{1}\right]} \approx \frac{k_{R I S C}}{k_{r}^{S}+k_{n r}^{S}+k_{I S C}} . \\
{\left[\mathrm{T}_{1}\right] \approx A_{T} \exp \left\{-\left[k_{r}^{T}+k_{n r}^{T}+\left(1-\frac{k_{R I S C}}{k_{r}^{S}+k_{n r}^{S}+k_{I S C}}\right) k_{R I S C}\right] t\right\}=A_{T} \exp \left(-k_{d} t\right),}
\end{gathered}
$$

where $A_{T}$ is a pre-exponential factor for the delayed decay component of the triplet excitons. Now, $k_{d}$ can be approximated as,

$$
k_{d} \approx k_{r}^{T}+k_{n r}^{T}+\left(1-\frac{k_{R I S C}}{k_{r}^{S}+k_{n r}^{S}+k_{I S C}}\right) k_{R I S C} .
$$

For this approximation, $k_{r}^{S}+k_{n r}^{S}+k_{I S C} \gg k_{R I S C}$ remains a necessary assumption to achieve the restriction condition of $\left[\mathrm{T}_{1}\right] \gg\left[\mathrm{S}_{1}\right]$. However, this assumption is valid in general as we explained previously. Invoking the SSA leads to a reduction in the number of required assumptions for the analysis of the kinetics of organic TADF materials.

The total decay efficiency of singlet excitons generated by photoexcitation is the sum of $\Phi_{r}^{S}, \Phi_{n r}^{S}$, and $\Phi_{I S C}$ (for the distribution of singlet exciton, the ISC/RISC cycles are not considered because $k^{S}$ was approximated as $k_{p}$ in this section by Eq. 3.5, i.e., $\Phi_{r}^{S} \approx \Phi_{P F}$ ), and the decay efficiency of triplet excitons that results from an ISC process is the sum of $\Phi_{r}^{T}, \Phi_{n r}^{T}$, and $\Phi_{R I S C}$, which are given by

$$
\begin{gathered}
\Phi_{r}^{S}+\Phi_{n r}^{S}+\Phi_{I S C}=1 \\
\Phi_{r}^{T}+\Phi_{n r}^{T}+\Phi_{R I S C}=1
\end{gathered}
$$

As a result of $k_{r}^{S}+k_{n r}^{S}+k_{I S C} \gg k_{R I S C}$, the RISC process is rate determining for the decay of $\mathrm{T}_{1}$ excitons via the $\mathrm{S}_{1}$ state while the $\mathrm{S}_{1}$ excitons generated by the RISC process rapidly decay to the $\mathrm{S}_{0}$ state or return back to the $\mathrm{T}_{1}$ state according to Eq. 3.10. In other words, the $\mathrm{T}_{1}$ excitons return to $\mathrm{T}_{1}$ state with a certain probability after ISC/RISC cycling. In this case, the efficiencies shown in Eq. 3.11 should be modified to take ISC/RISC cycling explicitly into account by using overall efficiencies (OEs), which correspond to the distributed exciton ratio between the $\mathrm{S}_{1}$ and $\mathrm{T}_{1}$ populations under the SSA via ISC/RISC cycles; these are $\Phi_{r}^{T}{ }^{O E}>\Phi_{r}^{T}, \Phi_{n r}^{T}{ }^{O E}>\Phi_{n r}^{T}$, and $\Phi_{R I S C}{ }^{O E}<\Phi_{R I S C}$ (see section 4 in supporting information for the detailed relationship between efficiencies and OEs). From these, $\Phi_{I S C}$ can be divided into $\Phi_{r}^{T^{O E}}, \Phi_{n r}^{T}{ }^{O E}$, and $\Phi_{R I S C}{ }^{O E}$, and the total efficiency is given by

$$
\Phi_{r}^{T^{O E}}+\Phi_{n r}^{T}{ }^{O E}+\Phi_{R I S C}{ }^{O E}=1 .
$$

The fraction of $\mathrm{T}_{1}$ exciton decays via $\mathrm{S}_{1}\left(\Phi_{I S C} \Phi_{R I S C}{ }^{O E}\right)$ can be divided to occur either radiatively $\left(\Phi_{r}^{S}\right)$ or nonradiatively $\left(\Phi_{n r}^{S}\right)$, because $\Phi_{R I S C}{ }^{O E}$ encompasses the exciton ratio after considering ISC/RISC cycling. The delayed fluorescence $\left(\Phi_{D F}\right)$ can now be formulated as a function of the radiative fraction to the total efficiency, $\Phi_{r}^{S}+\Phi_{n r}^{S}$.

$$
\Phi_{D F}=\Phi_{D E} R_{D E}^{D F}=\Phi_{I S C} \Phi_{R I S C}{ }^{O E} \frac{\Phi_{r}^{S}}{\Phi_{r}^{S}+\Phi_{n r}^{S}}=\Phi_{I S C} \Phi_{R I S C}{ }^{O E} \frac{\Phi_{r}^{S}}{1-\Phi_{I S C}}
$$

where $\Phi_{D E}$ are the quantum efficiency of phosphorescence and delayed emission, which are the sum of $\Phi_{D F}$ and phosphorescence $\left(\Phi_{P h o s}\right), \Phi_{D E}=\Phi_{D F}+\Phi_{p h o s} . R_{D E}^{D F}$ is the ratio of the delayed fluorescence component of the 
delayed emission $\left(\Phi_{D F} / \Phi_{D E}\right)$. On the other hand, the fraction of radiative decay from $\mathrm{T}_{1}\left(\Phi_{I S C} \Phi_{r}^{T^{O E}}\right)$ corresponds to $\Phi_{P h o s}$, as shown in Eq. 3.14.

$$
\Phi_{I S C} \Phi_{r}^{T}=\Phi_{P h o s}=\Phi_{P L Q Y}-\Phi_{P F}-\Phi_{D F}=\Phi_{D E}\left(1-R_{D E}^{D F}\right),
$$

In the three-state analysis, the lifetimes of TADF and phosphorescence are exactly the same since they occur from the same origin of the $\mathrm{T}_{1}$ state (see Eq. 2.20). Therefore, both $\Phi_{D F}$ and $\Phi_{P h o s}$ contribute to the delayed emission $\left(\Phi_{D E}\right)$. The total PL quantum yield $\left(\Phi_{P L Q Y}\right)$ is the sum of $\Phi_{r}^{S}$ and $\Phi_{D E}$. Based on the above analysis, all of the efficiencies related to the TADF process are presented in Eqs. 3.15-3.20.

$$
\begin{aligned}
& \Phi_{r}^{S}=\frac{k_{r}^{S}}{k_{r}^{S}+k_{n r}^{S}+k_{I S C}}=\frac{k_{r}^{S}}{k_{p}} . \\
& \Phi_{n r}^{S}=1-\Phi_{r}^{S}-\Phi_{I S C}=\frac{k_{n r}^{S}}{k_{r}^{S}+k_{n r}^{S}+k_{I S C}}=\frac{k_{n r}^{S}}{k_{p}} . \\
& \Phi_{I S C}=\frac{k_{I S C}}{k_{r}^{S}+k_{n r}^{S}+k_{I S C}}=\frac{k_{I S C}}{k_{p}} . \\
& \Phi_{r}^{T^{O E}}=\frac{\Phi_{\text {Phos }}}{\Phi_{I S C}}=\frac{k_{r}^{T}}{k_{r}^{T}+k_{n r}^{T}+\left(1-\Phi_{I S C}\right) k_{R I S C}}=\frac{k_{r}^{T}}{k_{d}} . \\
& \Phi_{n r}^{T}{ }^{O E}=\frac{k_{n r}^{T}}{k_{r}^{T}+k_{n r}^{T}+\left(1-\Phi_{I S C}\right) k_{R I S C}}=\frac{k_{n r}^{T}}{k_{d}} . \\
& \Phi_{R I S C}{ }^{O E}=\frac{\Phi_{D F}\left(1-\Phi_{I S C}\right)}{\Phi_{r}^{S} \Phi_{I S C}}=\frac{\left(1-\Phi_{I S C}\right) k_{R I S C}}{k_{r}^{T}+k_{n r}^{T}+\left(1-\Phi_{I S C}\right) k_{R I S C}}=\frac{\left(1-\Phi_{I S C}\right) k_{R I S C}}{k_{d}} .
\end{aligned}
$$

The corresponding rate constants are thus described by Eqs. 3.21-3.26.

$$
\begin{gathered}
k_{r}^{S}=k_{p} \Phi_{r}^{S} . \\
k_{n r}^{S}=k_{p} \Phi_{n r}^{S}=k_{p}\left(1-\Phi_{r}^{S}-\Phi_{I S C}\right) . \\
k_{I S C}=k_{p} \Phi_{I S C} . \\
k_{r}^{T}=k_{d} \Phi_{r}^{T^{O E}}=k_{d} \frac{\Phi_{P h o s}}{\Phi_{I S C}}=k_{d} \frac{\Phi_{D E}\left(1-R_{D E}^{D F}\right)}{\Phi_{I S C}} . \\
k_{n r}^{T}=k_{d}-\left(1-\Phi_{I S C}\right) k_{R I S C}-k_{r}^{T} . \\
k_{R I S C}=k_{R I S C} \frac{\Phi_{R I S C} O E}{1-\Phi_{I S C}}=\frac{k_{p} k_{d}}{k_{I S C}} \frac{\Phi_{D F}}{\Phi_{r}^{S}}=\frac{k_{d} \Phi_{D E} R_{D E}^{D F}}{\Phi_{r}^{S} \Phi_{I S C}} .
\end{gathered}
$$

As explained above, we obtained these rate equations with essentially no assumptions; they are nearly identical to those described in Ref 7. The value of $R_{D E}^{D F}$ can be obtained by fitting the delayed emission spectrum with the prompt fluorescence and phosphorescence spectra to provide the contribution of the phosphorescence to the delayed emission. However, we note that these equations still require $\Phi_{I S C}$ to be known. We can employ $\Phi_{n r}^{S}=0$ or $\Phi_{n r}^{T}=0$ as the limiting conditions to determine $\Phi_{I S C}$. This method should be applicable for most TADF materials; however, it should be noted that the model employs the approximation of $k^{S} \approx k_{p}$ in this section, which introduces a degree of uncertainty to the estimated rate constants, thus reducing their accuracy. 


\section{Reevaluation of rate equation using assumption of $\Phi_{n r}^{S}=0$ or $\Phi_{n r}^{T}=0$}

As it is difficult to measure directly $k_{I S C}$, in most of the literature the rate equations for TADF materials have been estimated using one of the assumptions of $\Phi_{n r}^{S}=0$ or $\Phi_{n r}^{T}=0$. The previously reported equations have been used indiscriminately to analyze not only highly emissive TADF materials but also poorly emissive materials, despite the inappropriateness of these models to handle the latter given their implicit assumptions. The derived rate constants must therefore be evaluated skeptically. The equations provided in the previous section using the SSA have an upward compatibility with previously reported models, especially those of Goushi-Masui and Dias, which have been often employed in the literature. ${ }^{8,10}$ When we employ $\Phi_{n r}^{S}=0$ or $\Phi_{n r}^{T}=0$ as a limiting condition to obtain $\Phi_{I S C}$, our equation using SSA leads to the same rate equations as those discussed in Refs 8 and $10\left(\Phi_{P h o s} \approx 0\right)$, respectively, shown here as Eqs. 4.1 and 4.2.

$$
\begin{gathered}
\Phi_{I S C}^{n r S=0}=1-\Phi_{P F} . \\
\Phi_{I S C}^{n r T=0}=\frac{\Phi_{D E}-\Phi_{P h o s}\left(1-\Phi_{P F}\right)}{\Phi_{P F}+\Phi_{D E}-\Phi_{P h o s}} .
\end{gathered}
$$

Where $\Phi_{I S C}^{n r S=0}$ and $\Phi_{I S C}^{n r T=0}$ correspond to the maximum and minimum values of $\Phi_{I S C}$, respectively. When $\Phi_{r}^{T} \approx 0$ (this approximation holds for organic TADF emitter behavior at around room temperature), the average $k_{I S C}$ and $k_{R I S C}$ values within the range between these limiting conditions $\left(k_{I S C}^{A v g .}\right.$ and $k_{R I S C}^{A v g}$, respectively) are provided as follows.

$$
\begin{aligned}
& k_{I S C}^{A v g .}=\frac{k_{p}}{2} \cdot \frac{\left[\Phi_{P L Q Y}\left(1-\Phi_{P F}\right)+\Phi_{D F}\right] \pm\left[\Phi_{P L Q Y}\left(1-\Phi_{P F}\right)-\Phi_{D F}\right]}{\Phi_{P L Q Y}} . \\
& k_{R I S C}^{A v g .}=\frac{k_{d}}{2} \cdot \frac{\left[\Phi_{P L Q Y}\left(1-\Phi_{P F}\right)+\Phi_{D F}\right] \pm\left[\Phi_{P L Q Y}\left(1-\Phi_{P F}\right)-\Phi_{D F}\right]}{\Phi_{P F}\left(1-\Phi_{P F}\right)} .
\end{aligned}
$$

The maximum and minimum values of $k_{I S C}^{A v g}$ are the values for the limiting conditions of $\Phi_{n r}^{S}=0$ and $\Phi_{n r}^{T}=0$, respectively, while the maximum and minimum values of $k_{R I S C}^{A v g}$ are the values for the limiting conditions of $\Phi_{n r}^{T}=0$ and $\Phi_{n r}^{S}=0$, respectively.

We analysed the range of $k_{R I S C}$ values of the three-state system by using Eq. 4.4. Figure 4a shows the plot of $k_{R I S C}^{A v g .}$ for the delayed emission ratio as a function of PLQY. The maximum and minimum values of the ranges are the values for the limiting conditions of $\Phi_{n r}^{T}=0$ and $\Phi_{n r}^{S}=0$, respectively. The plots of blue circles, green triangles, and red squares correspond to the respective values of $0.9,0.5$, and 0.1 for $\Phi_{D F}$. The prompt and delayed emission lifetimes $\left(\tau_{p}\right.$ and $\left.\tau_{d}\right)$ were fixed at $20 \mathrm{~ns}$ and $20 \mu \mathrm{s}$, which are representative values observed for organic TADF emitters. This plot reveals several important points: (1) the ratio of the delayed emission component significantly affects the magnitude of $k_{\text {RISC }}$. When the emission decay has only a small contribution from the delayed component, $k_{\text {RISC }}$ is not only slow but remains slow even if the material shows a high PLQY; (2) When the PLQY of TADF materials is not very high, i.e., less than 0.8 , there is a larger range of accessible $k_{\text {RISC }}$ values within the limiting conditions, regardless of the magnitude of $\Phi_{D E}$. Similar relationships also exist with respect to $k_{I S C}$. It is important to note that the assumption of $\Phi_{n r}^{S}=0$ results in both an underestimation of $k_{R I S C}$ and an overestimation of $k_{I S C}$ while the assumption of $\Phi_{n r}^{T}=0$ results in both an overestimation of $k_{R I S C}$ and an underestimation of $k_{I S C}$. The estimated 
$k_{R I S C}$ values do not change as a result of changes to the PLQY when the assumption of $\Phi_{n r}^{T}=0$ is employed. This in turn creates problems when this assumption is applied to the poorly emissive TADF materials. Especially for inefficient emitters $\left(\Phi_{P L Q Y}<0.1\right)$, the values of $k_{R I S C}$ under the assumption of $\Phi_{n r}^{S}=0$ or $\Phi_{n r}^{T}=0$ have been estimated with one order magnitude higher error depending on the ratio of $\Phi_{P F}$ and $\Phi_{D F}$ (Figure $4 \mathrm{~b}$ ). Further, despite when materials have high PLQY $\left(\Phi_{P L Q Y}>0.9\right)$, the difference between $k_{R I S C}^{n r S=0}$ and $k_{R I S C}^{n r T=0}$ can be more than double than the $\Phi_{P F} / \Phi_{D F}$ ratio. In the supporting information, several equations are provided to convert $k_{I S C}$ and $k_{R I S C}$ using a limiting condition to another condition or average rate constants, e.g. these equation make possible to interconvert the rate constants defined with the models of either Goushi-Masui or Dias and our models. When the $k_{p}$ and $k_{d}$ are each provided in the literature along with $\Phi_{P L Q Y}, \Phi_{P F}$, and $\Phi_{D F}$, Eqs. 4.2 and 4.3 allow direct extrapolation of the range of accessible values of $k_{I S C}$ and $k_{R I S C}$, and make possible the comparison between the value estimated using the different models and thus assess the possible range of values these rate constants can attain.

Recently, the importance of the role of intermediate triplet excited states in facilitating RISC processes, aided by spin-vibronic coupling has been elucidated..$^{25-28}$ Thus, we often find the delayed component of TADF shows biexponential decay. Naito et al. demonstrated the direct fitting of the TR PL decay by using the rate constants as a fitting parameter within a four-state model. ${ }^{26}$ Very recently, we also reported a rate analysis based on a four-state system consisting of $S_{1}, T_{1}, T_{n}$, and $S_{0}$, where $T_{n}$ is a triplet state of intermediate energy between $S_{1}$ and $T_{1}{ }^{29}$ The rate equations for the four-state system implicate that RISC proceeds via transient population of the intermediate $T_{n}$ state. However, it is difficult to derive the exact rate equations for the four-state analysis without invoking several a priori assumptions. Such a four-state system can also be modeled by using the SSA in a similar manner as we have described for the threestate system (see section 10 in supporting information). There are two required assumptions: the direct ISC/RISC process between the $S_{1}$ and $T_{1}$ states and the direct radiative/nonradiative processes from $T_{n}$ to $S_{0}$ be both forbidden, which are related to El-Sayed's and Kasha's rules, respectively. ${ }^{30,31}$ This situation occurs when $\mathrm{S}_{1}$ and $\mathrm{T}_{1}$ involve orbitals of the same orbital type and thus the corresponding ISC/RISC rate constants are negligibly small. Using a similar approach to that employed for the three-state system, we can obtain the rate equations that describe all of the rate constants, $k_{r}^{S}, k_{n r}^{S}, k_{I S C}, k_{R I S C}, k_{I C}^{T}, k_{R I C}^{T}$, and $k_{n r}^{T}$. Here, $k_{I C}^{T}$ and $k_{R I C}^{T}$ are the forward (IC) and reverse internal conversion (RIC) rate constants between $\mathrm{T}_{\mathrm{n}}$ and $\mathrm{T}_{1}$. We again confront the problem of determining $\Phi_{I S C}$. As explained in the previous section, we wish to avoid invoking the assumption that $\Phi_{n r}^{S}=0$ or $\Phi_{n r}^{T}=0$ in order to estimate $\Phi_{I S C}$. Therefore, we propose to determine $\Phi_{I S C}$ using, again, the average rate constants for the ISC, RISC, IC and RIC processes within a range of accessible values as an alternative, assuming $\Phi_{n r}^{S}=0$ or $\Phi_{n r}^{T}=0$. When $\Phi_{r}^{T} \approx 0$ is employed as a likely limiting condition, in an analogous manner to our previous analysis, $k_{I S C}, k_{R I S C}, k_{I C}$ and $k_{R I C}$ values within these conditions are given by Eqs. 4.5-4.8.

$$
\begin{gathered}
k_{I S C}^{A v g .}=\frac{k_{p}\left[2 \Phi_{P L Q Y}-\Phi_{P F}\left(1+\Phi_{P L Q Y}\right) \pm \Phi_{P F}\left(1-\Phi_{P L Q Y}\right)\right]}{2 \Phi_{P L Q Y}} . \\
k_{R I S C}^{A v g .}=\frac{k_{d 1} \Phi_{D E 1}\left[2 \Phi_{P L Q Y}-\Phi_{P F}\left(1+\Phi_{P L Q Y}\right) \pm \Phi_{P F}\left(1-\Phi_{P L Q Y}\right)\right]}{2 \Phi_{P F}\left(1-\Phi_{P F}\right)\left(\Phi_{P L Q Y}-\Phi_{P F}\right)} .
\end{gathered}
$$




$$
\begin{gathered}
k_{I C}^{A v g .}=k_{d 1}-\frac{k_{d 1} \Phi_{D E 1}\left[1+\Phi_{P L Q Y}-2 \Phi_{P F} \pm\left(1-\Phi_{P L Q Y}\right)\right]}{2\left(1-\Phi_{P F}\right)\left(\Phi_{P L Q Y}-\Phi_{P F}\right)} . \\
k_{R I C}^{A v g .}=\frac{k_{d 2}\left[2 \Phi_{D E 2}\left(1-\Phi_{P F}\right)+\Phi_{D E 1}\left(1-\Phi_{P L Q Y}\right) \pm \Phi_{D E 1}\left(1-\Phi_{P L Q Y}\right)\right]}{2 \Phi_{D E 1}\left(1-\Phi_{P F}-\Phi_{D E 1}\right)} .
\end{gathered}
$$

\section{Derivation of exact rate equations for three-state system containing $\boldsymbol{k}_{I S C}$.}

In section 2, we derived the exact equation to model the experimental bi-exponential emission decay of TADF materials. In section 3, we also provided a solution to determine the rate equations while minimizing the assumptions made. Though these rate equations provide several important insights, they are still based on the approximation of $k^{S} \approx k_{p}$. Here, we show that this approximation need not be invoked and derive the exact rate equations. In so doing, we aim to eliminate the confusion caused by implicating this approximation.

Here, we focus on the exponential decay of $\left[\mathrm{T}_{1}\right]$ to derive $k_{\mathrm{ISC}}$. In section 3 , we obtained the ratio of $\left[\mathrm{S}_{1}\right] /\left[\mathrm{T}_{1}\right]$ by applying the SSA to Eq. 2.1, but the exact value of $\left[\mathrm{S}_{1}\right] /\left[\mathrm{T}_{1}\right]$ within the delayed emission regime can be provided from the exact population decay equations of Eqs. 2.18 and 2.19 .

$$
\frac{\left[\mathrm{S}_{1}\right]}{\left[\mathrm{T}_{1}\right]}=\frac{k_{p}-k^{S}}{k_{I S C}}=\frac{k_{R I S C}}{k^{S}-k_{d}} .
$$

Therefore, Eq. 3.6 can be integrated and the evolution of $\left[\mathrm{T}_{1}\right]$ as a function of time becomes,

$$
\left[\mathrm{T}_{1}\right]=A_{d}^{T} \exp \left\{-\left[k_{r}^{T}+k_{n r}^{T}+\left(1-\frac{k_{I S C}}{k^{S}-k_{d}}\right) k_{R I S C}\right] t\right\}=\frac{\left[\mathrm{S}_{1}\right]_{t=0} k_{I S C}}{k_{p}-k_{d}} \exp \left(-k_{d} t\right),
$$

where $A_{d}^{T}$ is a preexponential factor of the delayed decay of triplet excitons. Therefore, $k_{d}$ can be written by,

$$
k_{d}=k_{r}^{T}+k_{n r}^{T}+\left(1-\frac{k_{I S C}}{k^{S}-k_{d}}\right) k_{R I S C}
$$

From the relationship of Eqs. 2.8 and 5.3, $k_{p}$ can be written by

$$
k_{p}=k_{r}^{S}+k_{n r}^{S}+\left(1+\frac{k_{R I S C}}{k^{S}-k_{d}}\right) k_{I S C} .
$$

Surprisingly, Eq. 5.4 implies that $k_{I S C}$ is slightly accelerated by the presence of multiple ISC/RISC cycles, and the resulting relationship of the observed decay rate of $S_{1}\left(k_{p}\right)$ is larger than the pure decay rate of $S_{1}\left(k^{S}\right)$, in spite of increasing the singlet exciton population due to the upconversion from a $\mathrm{T}_{1}$ state. The relationship $k_{p}>k^{S}$ was mentioned also in section 2. This phenomenon would be the result of the inflow from $\mathrm{T}_{1}$. From the relationship of Eqs. 2.5, 5.3 and 5.4, the overall efficiency of ISC and RISC $\left(\Phi_{I S C}{ }^{O E}, \Phi_{R I S C}{ }^{O E}\right)$ can be written as,

$$
\begin{gathered}
\Phi_{I S C}{ }^{O E}=\frac{\left(1+\frac{k_{R I S C}}{k^{S}-k_{d}}\right) k_{I S C}}{k_{p}}=\frac{k_{I S C}+k_{p}-k^{S}}{k_{p}}, \\
\Phi_{R I S C}{ }^{O E}=\frac{\left(1-\frac{k_{I S C}}{k^{S}-k_{d}}\right) k_{R I S C}}{k_{d}}=\frac{k_{R I S C}-k_{p}+k^{S}}{k_{d}} .
\end{gathered}
$$

Because $\Phi_{D F}$ can be explained as the overall emission efficiency from $S_{1}$ via $T_{1}$, it can be defined as, 


$$
\Phi_{D F}=\Phi_{D E} R_{D E}^{D F}=\Phi_{I S C}{ }^{O E} \Phi_{R I S C}{ }^{O E} \frac{\Phi_{P F}}{1-\Phi_{I S C}{ }^{O E}}=\frac{k_{r}^{S}}{k^{S}-k_{I S C}} \Phi_{I S C}{ }^{O E} \Phi_{R I S C}{ }^{O E},
$$

where $\Phi_{P F}$ corresponds to the overall quantum efficiency of the radiative decay from excitons populated directly at $\mathrm{S}_{1}$. Note that the generated $\mathrm{S}_{1}$ excitons via $\mathrm{T}_{1}$ described as $\Phi_{I S C}{ }^{O E}{ } \Phi_{R I S C}{ }^{O E}$ decay to $\mathrm{S}_{0}$ both radiatively or nonradiatively because $\Phi_{I S C}{ }^{O E} \Phi_{R I S C}{ }^{O E}$ is the final distributed exciton ratio of $\mathrm{S}_{1}$ via $\mathrm{T}_{1}$, considering the ISC/RISC cycles. They should not distribute to $\mathrm{T}_{1}$ anymore. From Eq. 5.7, the quadratic equation for $k_{I S C}$ can be obtained as

$$
\begin{gathered}
k_{I S C}=\frac{-b-\sqrt{b^{2}-4 a c}}{2 a}, \\
a=k_{d} \frac{\Phi_{D E}}{\Phi_{P F}} R_{D E}^{D F}-k_{p}+k^{S}, \\
b=\left(k_{p}-k^{S}\right)\left(k^{S}-k_{p}-k_{d}\right)-a k^{S}, \\
c=\left(k_{p}-k^{S}\right)^{2}\left(k^{S}-k_{d}\right) .
\end{gathered}
$$

As $k_{I S C}$ should be smaller than $k^{S}$, the value is uniquely determined as described in Eq. 5.8. When we can approximate that the delayed emission does not contain phosphorescence $\left(R_{D E}^{D F} \approx 1\right)$, the value of $a$ becomes 0 and the equation for $k_{I S C}$ can be rewritten more simply as,

$$
k_{I S C}=\frac{\left(k_{p}-k^{S}\right)\left(k^{S}-k_{d}\right)}{k_{d}+k_{p}-k^{S}} .
$$

$k_{R I S C}$ can be obtained from the relationship shown in Eq. 5.13, which is also found in Eq. 2.5. The equation for $k_{R I S C}$ is provided as Eq. 5.14 with the approximation of $R_{D E}^{D F} \approx 1$.

$$
\begin{gathered}
k_{R I S C}=\frac{\left(k_{p}-k^{S}\right)\left(k^{S}-k_{d}\right)}{k_{I S C}} . \\
k_{R I S C}=k_{d}+k_{p}-k^{S} .
\end{gathered}
$$

From Eq. 2.20, the ratio of the pre-exponential factor, $A_{d} / A_{p}$, associated with the prompt and delayed components ( $A_{p}$ and $A_{d}$, respectively) can be written by,

$$
\frac{A_{d}}{A_{p}}=\frac{\left(k_{p}-k^{S}\right) \Phi_{r}^{S}+k_{I S C} \Phi_{r}^{T}}{\left(k^{S}-k_{d}\right) \Phi_{r}^{S}-k_{I S C} \Phi_{r}^{T}}
$$

Eqs. 2.22, 3.2, 3.3, and 5.15 provide an exact solution for the singlet decay rate $\left(k^{S}\right)$ as,

$$
k^{S}=k_{p}-k_{d} \frac{\Phi_{D E}}{\Phi_{P F}}+k_{I S C} \frac{\Phi_{r}^{T}}{\Phi_{r}^{S}}
$$

When $R_{D E}^{D F} \approx 1, k^{S}$ is simplified as the phosphorescence related term vanishes as Eq. 5.17.

$$
k^{S}=k_{p}-k_{d} \frac{\Phi_{D E}}{\Phi_{P F}}
$$

The rate constants for non-radiative decay from $S_{1}$ and $T_{1}$ can be extracted from Eq. 2.8 without invoking the simplification of $R_{D E}^{D F} \approx 1$ as,

$$
\begin{gathered}
k_{n r}^{S}=k^{S}-k_{r}^{S}-k_{I S C}, \\
k_{n r}^{T}=k^{T}-k_{R I S C}-k_{r}^{T}=k_{d}+k_{p}-k^{S}-k_{R I S C}-k_{r}^{T} .
\end{gathered}
$$


When data is collected at $300 \mathrm{~K}$ or in solution state, it is not unusual to assume the observed emission does not contain phosphorescence $\left(\Phi_{P h o s} \approx 0\right)$, i.e., $R_{D E}^{D F} \approx 1$. However, $k_{n r}^{T}$ is always 0 when approximated as $R_{D E}^{D F} \approx 1$ because of Eqs. 5.14 and 5.19. As a result, the rate constants for $k_{n r}^{S}, k_{I S C}$, and $k_{R I S C}$ under the approximation of $R_{D E}^{D F} \approx 1$ can be written in terms of decay rates and efficiencies for prompt and delayed component as shown in Eqs. 5.20-5.22. In this case, it should be noted that $k_{R I S C}$ is obtained as a maximum for these possible values.

$$
\begin{gathered}
k_{n r}^{S}=k_{p} \frac{\Phi_{P F}}{\Phi_{P L Q Y}}\left(1-\Phi_{P L Q Y}\right), \\
k_{I S C}=k_{p} \frac{\Phi_{D F}}{\Phi_{P L Q Y}}-k_{d} \frac{\Phi_{D F}}{\Phi_{P F}} . \\
k_{R I S C}=k_{d} \frac{\Phi_{P L Q Y}}{\Phi_{P F}} .
\end{gathered}
$$

Interestingly, the rate equation for $k_{R I S C}$ is exactly same with the model of Dias. The radiative decay rate $k_{r}^{S}$ is independently obtained by,

$$
k_{r}^{S}=k_{p} \Phi_{P F}
$$

Next, we provide the exact solution of the kinetics analysis for TADF of a three-state system without any assumptions and approximations. The relationship related to the phosphorescence is provided as,

$$
\Phi_{I S C}{ }^{O E} \Phi_{r}^{T}{ }^{O E}=\Phi_{P h o s}=\Phi_{P L Q Y}-\Phi_{P F}-\Phi_{D F}=\Phi_{D E}\left(1-R_{D E}^{D F}\right),
$$

From Eq. 5.24 the rate equation and radiative decay quantum efficiency for $\mathrm{T}_{1}$ excitons $\left(k_{r}^{T}\right.$ and $\left.\Phi_{r}^{T}\right)$ can be described as Eqs. 5.25 and 2.26.

$$
\begin{gathered}
k_{r}^{T}=\frac{k_{d} \Phi_{D E}\left(1-R_{D E}^{D F}\right)}{\Phi_{I S C}{ }^{O E}}=\frac{k_{p} k_{d} \Phi_{D E}\left(1-R_{D E}^{D F}\right)}{k_{I S C}+k_{p}-k^{S}} . \\
\Phi_{r}^{T}=\frac{k_{d} \Phi_{r}^{T}}{k^{T} \Phi_{I S C}^{O E}}=\frac{k_{p} k_{d} \Phi_{D E}\left(1-R_{D E}^{D F}\right)}{\left(k_{p}+k_{d}-k^{S}\right)\left(k_{I S C}+k_{p}-k^{S}\right)} .
\end{gathered}
$$

From the Eqs. 5.16 and 5.24 the exact $k^{S}$ can be obtained as a solution of the following cubic equation,

$$
\begin{gathered}
k^{S^{3}}+d k^{S^{2}}+e k^{S}+f=0 . \\
d=-\left[k_{I S C}+3 k_{p}+k_{d}\left(1-\frac{\Phi_{D E}}{\Phi_{P F}}\right)\right] . \\
e=\left[2 k_{I S C} k_{p}+3 k_{p}^{2}+\left(2 k_{p} k_{d}+k_{I S C} k_{d}\right)\left(1-\frac{\Phi_{D E}}{\Phi_{P F}}\right)-k_{d}{ }^{2} \frac{\Phi_{D E}}{\Phi_{P F}}-\frac{k_{I S C} k_{d} \Phi_{D E}\left(1-R_{D E}^{D F}\right)}{\Phi_{P F}}\right] . \\
f=-\left(k_{I S C}+k_{p}\right)\left(k_{p}+k_{d}\right)\left(k_{p}-k_{d} \frac{\Phi_{D E}}{\Phi_{P F}}\right) .
\end{gathered}
$$

Because $k^{S}$ should be smaller than $k_{p}$ and larger than $k_{I S C}\left(k_{I S C}<k^{S}<k_{p}\right)$, the solution to the cubic equation is uniquely determined using the Cardano-Tartaglia formula as,

$$
k^{S}=\frac{-1+i \sqrt{3}}{2} \times \sqrt[3]{-\frac{27 f+2 d^{3}-9 d e}{54}+\sqrt{\left(\frac{27 f+2 d^{3}-9 d e}{54}\right)^{2}+\left(\frac{3 e-d^{2}}{9}\right)^{3}}}
$$




$$
+\frac{-1-i \sqrt{3}}{2} \times \sqrt[3]{-\frac{27 f+2 d^{3}-9 d e}{54}-\sqrt{\left(\frac{27 f+2 d^{3}-9 d e}{54}\right)^{2}+\left(\frac{3 e-d^{2}}{9}\right)^{3}}}-\frac{1}{3} d
$$

Because the exact equations for $k_{I S C}$ and $k^{S}$ contain the terms of $k^{S}$ and $k_{I S C}$, respectively, a numerical analysis is required to obtain the exact rate. For example, $k_{I S C}$ at $R_{D E}^{D F} \approx 1$ can be provided to obtain $k^{S}$ and then estimated $k_{I S C}$ were evaluated to avoid the circular reference; a new $k_{I S C}$ value can be provided to minimize the difference between the given and estimated values. The $R_{D E}^{D F}$ is given as all rate constants are provided as $\geq 0$.

Here, we derived the exact rate equations for organic TADF materials based on the three-state model. It is noteworthy that the equations in this section considerably reduce the errors in the estimation of the rate constants compared with the previous methods without the requirement of additional experiments except the transient PL decay and PLQY measurements to obtain $k_{I S C}$. The equation for $k_{I S C}$ cannot be applied to the previously reported rate equations that use the approximation of $k^{S} \approx k_{p}$. We note here again, the emission lifetimes of TADF and phosphorescence are exactly the same as in the three-state system because both decays are related to the $\mathrm{T}_{1}$ decay; the ISC/RISC cycles behave as an exciton pool for both decays. Therefore, the delayed emission should always include both contributions from delayed fluorescence and phosphorescence at the fixed ratio $\left(R_{D E}^{D F}\right.$ and $\left.1-R_{D E}^{D F}\right)$ in the threestate model. In other words, when data contain three or more decays, the "exact" rate equation derived in this section should not be applied. For example, phosphorescence often can be found as an additional radiative decay distinct from TADF. In this case, the material should be analyzed by another modes, e.g., four-state model explained in SI of this paper. Because the rate equations describing the three-state system were derived considering the phosphorescence is intrinsic to the delayed emission, it is favorable to collect the emission decay data in the full-range of fluorescence and phosphorescence spectra, i.e., the decay data collected not only by a single wavelength measurement but also with multiple wavelength measurements. When the single wavelength measurement is employed, the measurement wavelength should be a wavelength around the intersection between the normalized fluorescence and phosphorescence spectra.

Finally, we re-estimated the rate constants for several TADF materials in the literature: $4 \mathrm{CzIPN} ;{ }^{27} 5 \mathrm{CzBN} ;{ }^{27,32}$ 3Cz2DPhCzBN; ${ }^{32}$ 5Cz-TRZ; ${ }^{33}$ TQ; $;{ }^{34}$ v-DABNA; ${ }^{35}$ TMCz-BO; ${ }^{36}$ TPAt-tFFO; ${ }^{11} \mathrm{Br}-3 \mathrm{PXZ}-\mathrm{XO} ;{ }^{37} \mathrm{DiKTa} ;{ }^{38}$ and MCzTXT. ${ }^{39}$ We undertook this analysis with the approximation of $R_{D E}^{D F} \approx 1$. The rate constant values listed in Table 1 contain the originally reported values from the literature, the values using the equations where the SSA is invoked with the general estimation method of $\Phi_{P F}$ and $\Phi_{D E}$ (SSA-1), the values using equations where the SSA is invoked with the corrected estimation method of $\Phi_{P F}$ and $\Phi_{D E}$ (SSA-2), and using the "exact" equations for the three-state model. For SSA-1 and SSA-2, the $k_{I S C}$ and $k_{R I S C}$ values are provided with the limiting conditions of $k_{n r}^{S}=0$ and $k_{n r}^{T}=$ 0 .

An evaluation of the results using these different models reveals that the literature reported $k_{R I S C}$ values and those using SSA-1 are similar. Most of the literature reported $k_{R I S C}$ values using the assumption of $k_{n r}^{S}=0$ and those using the exact value $\left(k_{n r}^{T}=0\right)$ show no difference; however, this is the result of a fortuitous cancellation of errors with the assumption of $k_{n r}^{S}=0$, the approximation of $k^{S} \approx k_{p}$, and the estimation method of $\Phi_{P F}$ and $\Phi_{D E}$. 
The $k_{R I S C}$ values of TPAt-tFFO, which is estimated with the assumption of $k_{n r}^{T}=0$, show relatively large differences depending on the model used. This is related to the estimation method of $\Phi_{P F}$ and $\Phi_{D E}$ and the use of the approximation via a Maclaurin expansion. For the $k_{R I S C}$ estimation, the SSA-2 with the condition of $k_{n r}^{T}=0$ showed good agreement with those using the exact equation. This is because both rate equations to determine $k_{R I S C}$ are exactly the same (see Eqs. 5.22 and S7.5) when approximating $R_{D E}^{D F} \approx 1$ and when using SSA with the limiting condition of $k_{n r}^{T}=0$. When TADF materials show very fast $\mathrm{T}_{1}-\mathrm{S}_{1}$ upconversion, i.e. $k_{p} \approx k_{d}$, will become available, the equations using the SSA cannot be applied, but there remains no restriction to the use of the exact equations. In addition, when using the wrong estimation of $\Phi_{P F}$ and $\Phi_{D E}$ by Eqs. S3.1 and S3.2 employed in many literature reports, $k_{I S C}$ tended to be overestimated; this can be found by comparing the $k_{I S C}$ between SSA-1 and SSA-2. To reduce the estimation error of the rate constants, it is important to use the corrected estimation method for $\Phi_{P F}$ and $\Phi_{D E}$. Further, Eqs. 5.21 and S6.4 provide the correction coefficient, $-k_{d} \Phi_{D F} / \Phi_{P F}$ for $k_{I S C}$ to exclude the affection of $k^{S} \approx k_{p}$. Thus, all derived equations help understand the spin-flip processes with the exact rate constants for reported TADF materials; the equations in the model with practically no assumptions allow the conversion of the rate constants obtained using the model of Goushi-Masui's to those using the model of Dias, and the correction coefficient between the model of Dias and exact model provides the exact rate constants.

As an additional finding, the estimated rate constants numerical analysis showed no change with the case of $R_{D E}^{D F} \approx 1$ for most of the materials explained above. Both $k_{r}^{T}$ and $k_{n r}^{T}$ are estimated as $\ll 1$. Therefore, the approximation of $R_{D E}^{D F} \approx 1$ should be reasonable. This finding is highly suggestive in the photophysics in three-state system. When there is the decay channel of $T_{1}$ via $S_{1}$, then direct decay from $T_{1}$ to $S_{0}$ is not available. To explain the direct decay of $\mathrm{T}_{1}$, a new model would be necessary to explain the materials that show the dual emission of TADF and room temperature phosphorescence. The four-state kinetics analysis explained in the SI would help to understand advanced function of emissive materials.

\section{Summary}

In this paper, we provided the exact decay curve equation for the TADF materials on the three-state system. The equation was derived from exact $S_{1}$ and $T_{1}$ population decays considering all exciton decay processes. The exact equations of exciton population decays help advance our understanding of the photophysics of not only TADF materials but also materials related to the three-state system of $S_{1}, T_{1}$, and $S_{0}$, because these equations were derived without employing any approximations and assume not particular preferential decay pathway. In addition, the equations to estimate the efficiencies for the prompt and delayed emission components $\left(\Phi_{P F}\right.$ and $\left.\Phi_{D E}\right)$ were corrected. The estimation method of efficiencies strongly influenced the estimated value of rate constants. Further, we demonstrated that it is possible to derive the rate equations with "practically" and "perfectly" no-assumptions. The rate equations are summarized in Table 2.

For the first method, we employed two approximations. One is $k^{S} \approx k_{p}$, which is commonly used, to analyse the prompt emission under the condition of $\left[\mathrm{S}_{1}\right] \gg\left[\mathrm{T}_{1}\right]\left(t \ll 1 / k_{p}\right)$. This approximation requires the assumption of 
$k_{r}^{S}+k_{n r}^{S}+k_{I S C} \gg k_{R I S C}$; however, this is satisfied because of the relationship of exo- and endothermic processes of $k_{I S C}$ and $k_{R I S C}$. The other one is the steady-state approximation of the $\mathrm{S}_{1}$ population to obtain the $\left[\mathrm{S}_{1}\right] /\left[\mathrm{T}_{1}\right]$ ratio at the delayed emission region of $\left[\mathrm{S}_{1}\right] \ll\left[\mathrm{T}_{1}\right]\left(t \gg 1 / k_{p}\right)$. This approximation also requires the assumption of $k_{r}^{S}+$ $k_{n r}^{S}+k_{I S C} \gg k_{R I S C}$. To analyse the experimental data, this method requires that the $\Phi_{I S C}$ must be provided by additional measurement; however, it is possible to employ $\Phi_{n r}^{S}=0$ or $\Phi_{n r}^{T}=0$ as not assumptions but as limiting conditions. This practically no-assumption method makes exchange these two assumptions used in the most of literatures being able to compare the reported values. This method has some margin of error; however, it should be noted that there are some inapplicable cases because of the approximations employed. When $\Phi_{I S C}$ is not provided, the rate constants should be reported as average values between both sets of limiting conditions. In addition, the rate equations for the TADF materials using a four-state system of $S_{0}, S_{1}, T_{n}$ and $T_{1}$ are derived in the supporting information.

We also derived the "exact" rate equations for the TADF materials on the three-state model. In this model, $\Phi_{I S C}$ can be estimated without any other additional measurements other than emission spectra, transient emission decay, and PLQY. This method requires the estimation of $\Phi_{\text {phos }}$, which can be obtained by spectral fitting or numerical analysis. However, there is difficult case because of the high accuracy of the equations. In this case, the equations allow to approximate $\Phi_{\text {phos }} \approx 0$, which can be applied to the data collected at temperatures where TADF is operational and is used in most of the literature reports. With the approximation of no phosphorescence contribution, the rate equations simplify and a consequence of this approximation within the model is that $\Phi_{n r}^{T}=0$; i.e. the rate constants of radiative and non-radiative decay from $\mathrm{T}_{1}$ are coupled. This method provides the most precise set of rate constants for TADF materials. Further, the exact equations reveal the presence of an important relationship on the photophysics; the singlet population decay is accelerated by the presence of a RISC process. By using either model reported here, we can derive the exact rate constants from reported values in the literature by analysing the data using the modesl of either GoushiMasui or Dias, which are the ones most commonly used. We believe the equations provided here will enable the universalization of the kinetics analysis of TADF materials and so lead to a better understanding of their photophysics, and ultimately better materials.

\section{Acknowledgements}

The work at Kyushu University was supported financially by the Program for Building Regional Innovation Ecosystems of the Ministry of Education, Culture, Sports, Science and Technology, Japan, JST ERATO Grant JPMJER1305, Japan, and JSPS Core-to-core Program, and Kyulux Inc. The work at the University of St Andrews was supported by the EPSRC (EP/P010482/1, EP/R035164/1). 


\section{References}

1 Wong, M. Y. \& Zysman-Colman, E. Purely organic thermally activated delayed fluorescence (TADF) materials for organic light-emitting diodes (OLEDs). Adv. Mater. 29, 1605444 (2017).

2 Yang, Z., Mao, Z., Xie, Z., Zhang, Y., Liu, S., Zhao, J., Xu, J., Chi, Z. \& Aldredb, M. P. Recent advances in organic thermally activated delayed fluorescence materials. Chem. Soc. Rev. 46, 915-1016 (2017).

3 Liu, Y., Li, C., Ren, Z., Yan, S. \& Bryce, M. R. All-organic thermally activated delayed fluorescence materials for organic light-emitting diodes. Nat. Rev. Mater. 3, 18020 (2018).

4 Bui, T.-T., Goubard, F., Ibrahim-Ouali, M., Gigmes, D. \& Dumur, F. Recent advances on organic blue thermally activated delayed fluorescence (TADF) emitters for organic light-emitting diodes (OLEDs). Beilstein J. Org. Chem. 14, 282-308 (2018).

5 Kirchhoff, J. R., Gamache Jr., R. E., Blaskie, M. W., Paggio, A. A. D., Lengel, R. K. \& McMillin D. R. Temperature dependence of luminescence from $\mathrm{Cu}(\mathrm{NN})_{2}{ }^{+}$systems in fluid solution. Evidence for the participation of two excited states. Inorg. Chem. 22, 2380-2384 (1983).

6 Zhang, Q., Li, B., Huang, S., Nomura, H., Tanaka, H. \& Adachi, C. Efficient blue organic light-emitting diodes employing thermally activated delayed fluorescence. Nat. Photonics 8, 326-332 (2012).

7 Goushi, K., Yoshida, K., Sato, K. \& Adachi C. Organic light-emitting diodes employing efficient reverse intersystem crossing for triplet-to-singlet state conversion. Nat. Photonics. 6, 253-258 (2012).

8 Masui, K., Nakanotani, H. \& Adachi, C. Analysis of exciton annihilation in high-efficiency sky-blue organic light-emitting diodes with thermally activated delayed fluorescence. Org. Electron. 14, 2721-2726 (2013).

9 Pan, K.-C., Li, S.-W., Ho, Y.-Y., Shiu, Y.-J., Tsai, W.-L., Jiao, M., Lee, W.-K., Wu, C.-C., Chung, C.-L., Chatterjee, T., Li, Y.-S. \& Wong, K.-T. Efficient and tunable thermally activated delayed fluorescence emitters having orientation-adjustable $\mathrm{CN}$-substituted pyridine and pyrimidine acceptor units. Adv. Funct. Mater. 26, 7560-7571 (2016).

10 Dias, F., Penfold, T. J. \& Monkman A. P. Photophysics of thermally activated delayed fluorescence molecules. Methods Appl. Fluoresc. 5, 012001 (2017).

11 Wada, Y., Nakagawa, H., Matsumoto, S., Wakisaka, Y. \& Kaji, H. Organic light emitters exhibiting very fast reverse intersystem crossing. Nat. Photonics 14, 643-649 (2020).

12 Hasse, N., Danos, A., Pflumm, C., Morherr, A., Stachelek, P., Mekic, A., Bütting, W. \& Monkman A. P. Kinetic Modeling of transient photoluminescence from thermally activated delayed fluorescence. J. Phys. Chem. C 122, 29173-29179 (2018).

13 Yurash, B., Nakanotani, H., Olivier, Y., Beljonne, D., Adachi, C. \& Nguyen T.-Q. Photoluminescence Quenching probes spin conversion and exciton dynamics in thermally activated delayed fluorescence materials. Adv. Mater. 31,1804490 (2019).

14 Vázquez, R. J., Yun, J. H., Muthike, A. K., Howell, M., Kim, H., Madu, I. K., Kim, T., Zimmerman, P., Lee, J. Y., \& Goodson III, T. New direct approach for determining the reverse intersystem crossing rate in organic thermally activated delayed fluorescent (TADF) emitters. J. Am. Chem. Soc. 142, 8074-8079 (2020). 
15 Notsuka, N., Nakanotani, H., Noda, H., Goushi, K. \& Adachi, C. Observation of nonradiative deactivation behavior from singlet and triplet states of thermally activated delayed fluorescence emitters in solution. J. Phys. Chem. Lett. 11, 562-566 (2020).

16 Scott, D. R. \& Maltenieks, O. Experimental method for determining the intersystem crossing rate constant from lowest excited singlet to lowest triplet state. J. Phys. Chem. 72, 3354-3356 (1968).

17 Carmichael, I. \& Hug, G. L. Triplet-triplet absorption spectra of organic molecules in condensed phase. J. Phys. Chem. Ref. Data 15, 1-250 (1986).

18 Berberan-Santos, M. N. \& Garcia, J. M. M. Unusually strong delayed fluorescence of C70. J. Am. Chem. Soc. 118, 9391-9394 (1996).

19 Baleizão, C. \& Berberan-Santos, M. N. Thermally activated delayed fluorescence as a cycling process between excited singlet and triplet states: Application to the fullerenes. J. Chem. Phys. 126, 204510 (2007).

20 Kobayashi, T., Kawate, D., Niwa, A., Nagase, T., Goushi, K., Adachi, C., \& Naito, H. Intersystem crossing rate in thermally activated delayed fluorescence emitters. Phys. Status Solidi A 217, 1900616 (2020).

21 Niwa, A., Takaki, K., Kobayashi, T., Nagase, T., Goushi, K., Adachi, C. \& Naito, H. Photoluminescence in a Thermally activated delayed fluorescence emitter for organic light-emitting diodes. J. Imaging Soc. Jpn. 55, 143148 (2016).

22 Kobayashi, T., Niwa, A., Haseyama, S., Takaki, K., Nagase, T., Goushi, K., Adachi, C. and Naito, H. Emission properties of thermally activated delayed fluorescence emitters: analysis based on a four-level model considering a higher triplet excited state. J. Photon. Energy 8, 032104 (2018).

23 Serevičius, T., Skaisgiris, R., Kreiza, G., Dodonova, J., Kazlauskas, K., Orentas, E., Tumkevičius, S., and Juršènas, S. TADF Parameters in the Solid State: An Easy Way to Draw Wrong Conclusions. J. Phys. Chem. A 125, 1637-1641 (2021).

24 Zhang, Q., Kuwabara, H., Potscavage, Jr., W. J., Huang, S., Hatae, Y., Shibata, T., and Adachi, C. AnthraquinoneBased Intramolecular Charge-Transfer Compounds: Computational Molecular Design, Thermally Activated Delayed Fluorescence, and Highly Efficient Red Electroluminescence. J. Am. Chem. Soc. 136, 18070-18081 (2014).

25 Etherington, M. K., Gibson, J., Higginbotham, H. F., Penfold, T. J. \& Monkman, A. P. Revealing the spinvibronic coupling mechanism of thermally activated delayed fluorescence. Nat. Commun. 7, 13680 (2016).

26 Kobayashi, T., Niwa, A., Takaki, K., Haseyama, S., Nagase, T., Goushi, K., Adachi, C. \& Naito H. Contributions of a higher triplet excited state to the emission properties of a thermally activated delayed-fluorescence emitter. Phys. Rev. Appl. 7, 034002 (2017).

27 Noda, H., Chen, X.-K., Nakanotani, H., Hosokai, T., Miyajima, M., Notsuka, N., Kashima, Y., Brédas, J.-L. \& Adachi, C. Critical role of intermediate electronic states for spin-flip processes in charge-transfer-type organic molecules with multiple donors and acceptors. Nat. Mater. 18, 1084-1090 (2019).

28 E. Zysman-Colman. Molecular designs offer fast exciton conversion. Nat. Photonics 14, 593-594 (2020). 
29 Tsuchiya, Y., Tsuji, K., Inada, K., Bencheikh, F., Geng, Y., Kwak, H. S., Mustard, T. J. L., Halls, M. D., Nakanotani, H. \& Adachi, C. Molecular design based on donor-weak donor scaffold for blue thermally-activated delayed fluorescence designed by combinatorial DFT calculations. Front. Chem. 8, 403 (2020).

30 El-Sayed, M. A. Spin-orbit coupling and the radiationless processes in nitrogen heterocyclics. J. Chem. Phys. 38, 2834-2838 (1963).

31 Kasha, M. Characterization of electronic transitions in complex molecules. Discuss. Faraday Soc. 9, 14-19 (1950).

32 Noda, H., Nakanotani, H., Adachi, C. Excited state engineering for efficient reverse intersystem crossing. Sci. $A d v .4$, eaa06910 (2018).

33 Cui, L.-S., Gillett, A. J., Zhang, S.-F., Ye, H., Liu, Y., Chen, X.-K., Lin, Z.-S., Evans, E. W., Myers, W. K., Ronson, T. K., Nakanotani. H., Reineke, S., Bredas, J.-L., Adachi, C. \& Friend R. H. Fast spin-flip enables efficient and stable organic electroluminescence from charge-transfer states. Nat. Photonics 14, 636-642 (2020).

34 Mamada, M., Inada, K., Komino, T., Potscavage Jr., W. J., Nakanotani, H., Adachi. C. Highly Efficient Thermally Activated Delayed Fluorescence from an Excited-State Intramolecular Proton Transfer System. ACS Cent. Sci. 3 , 769-777 (2017).

35 Kondo, Y., Yoshiura, K., Kitera, S., Nishi, H., Oda, S., Gotoh, H., Sasada, Y., Yanai, M. \& Hatakeyama, T. Nat. Photonics 13, 678-682 (2019).

36 Kim, J.-U., Park, I.-S., Chan. C.-Y., Tanaka, M., Tsuchiya, Y., Nakanotani. H. \& Adachi, C. Nanosecond-timescale delayed fluorescence molecule for deep-blue OLEDs with small efficiency rolloff. Nat. Commun. 11, 1765 (2020).

37 Aizawa, N., Harabuchi, Y., Maeda, S. \& Pu, Y.-J. Kinetic prediction of reverse intersystem crossing in organic donor-acceptor molecules. Nat. Commun. 11, 3909 (2020).

38 Hall, D., Suresh, S. M., dos Santos, P. L., Duda, E., Bagnich, S., Pershin, A., Rajamalli, P., Cordes, D. B., Slawin, A. M. Z., Beljonne, D., Köhler, A., Samuel, I. D. W., Olivier, Y., Zysman-Colman, E., Improving Processability and Efficiency of Resonant TADF Emitters: A Design Strategy, Adv. Opt. Mater. 8, 1901627 (2020).

39 Aizawa, N., Matsumoto, A., Yasuda, T., Thermal equilibration between singlet and triplet excited states in organic fluorophore for submicrosecond delayed fluorescence. Sci. Adv. 7, eabe5769 (2021). 


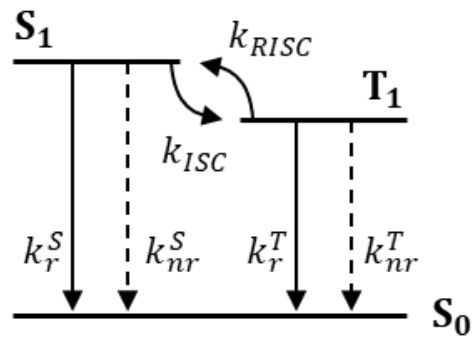

Figure 1. The scheme of photophysical process for three-state system.

(a)

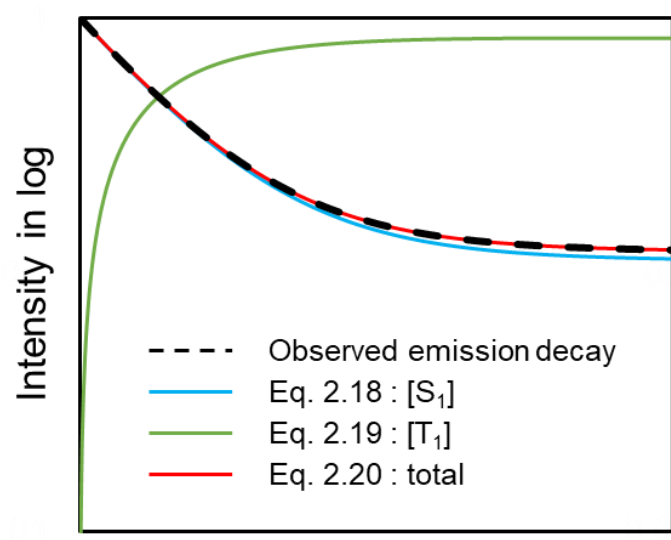

Time (ns) (b)

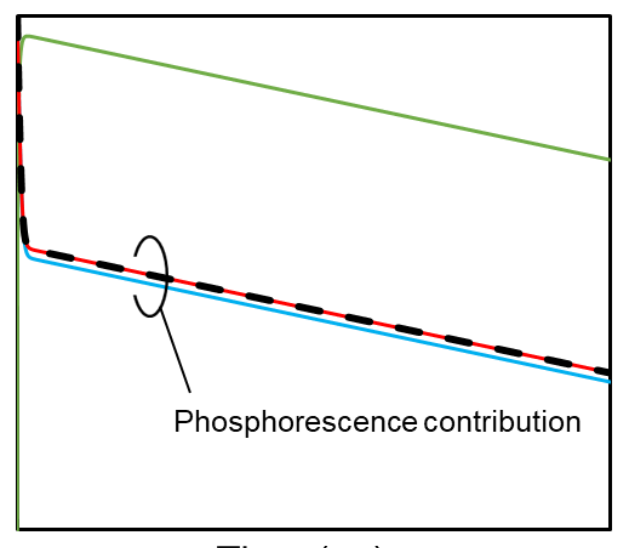

Time ( $\mu \mathrm{s})$

Figure 2. Emission decay curve and theoretical curves of Eqs. 2.18-2.20 within nano second time range (a) and milli second time range (b). the difference of total emission and $S_{1}$ population decays is related to the phosphorescence contribution.

(a)

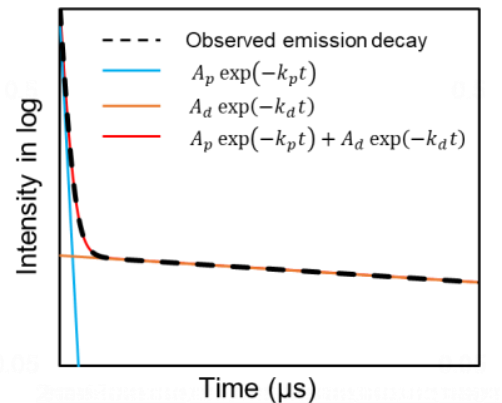

(b)

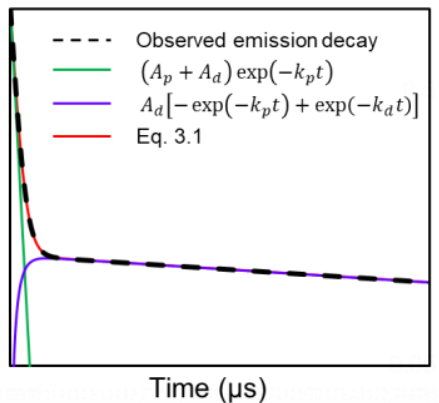

(c)

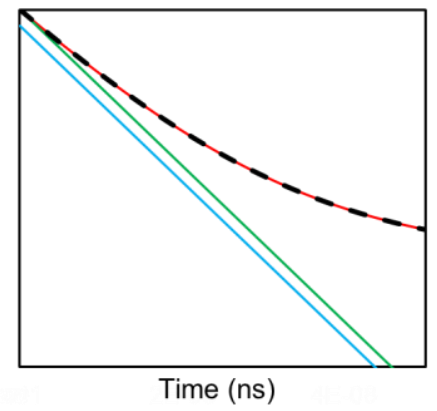

Figure 3. (a) Emission decay curve and biexponential fitting curves which are employed to estimate the prompt and delayed emission efficiency in general. (b) Corrected prompt and delayed component curves to provide exact emission efficiencies. (c) Closeup within nano second range to recognise difference of prompt components for general and corrected estimation method. 
(a)

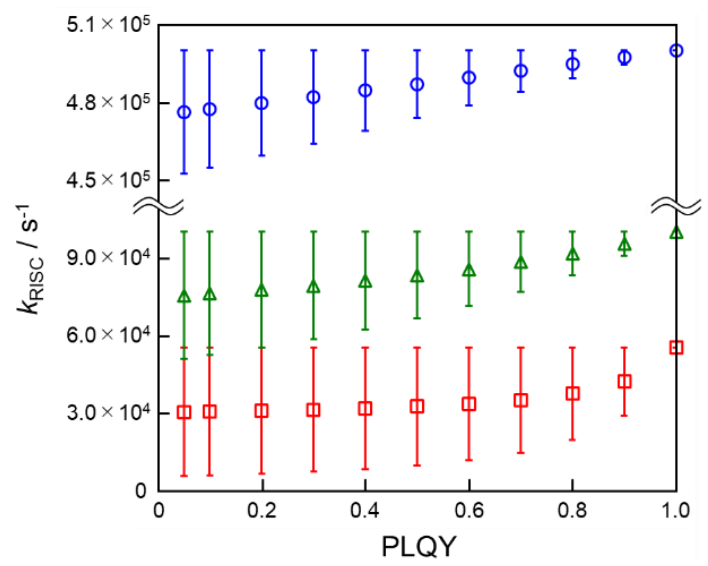

(b)

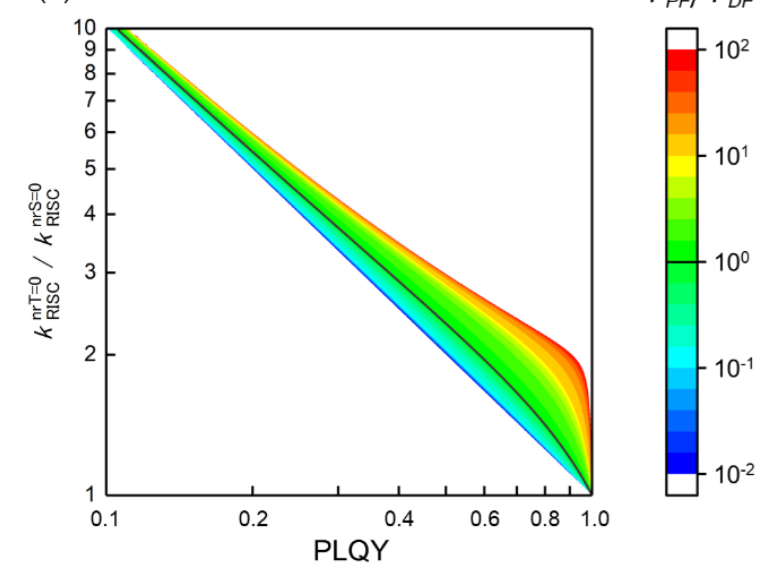

Figure 4. (a) Plot of $k_{R I S C}^{A v e}$ for the delayed emission ratio to PLQY; blue circle, $\Phi_{P F}: \Phi_{D F}=0.1: 0.9$; green triangle, $\Phi_{P F}: \Phi_{D F}=0.5: 0.5 ;$ red square, $\Phi_{P F}: \Phi_{D F}=0.9: 0.1 ; 1 / k_{p}, 20 \mathrm{~ns} ; 1 / k_{d}, 20 \mu \mathrm{s} ;$ plot was generated using Eq. 4.4. (b) Ratio of RISC rate constant between assuming $k_{n r}^{S}=0$ and $k_{n r}^{T}=0$ for each PLQY with color properties indicating the ratio of $\Phi_{P F}$ and $\Phi_{D F}$; solid black line, $\Phi_{P F}: \Phi_{D F}=1$; plot was generated by using Eq. 4.4. 
Table 1. Rate constants of interest TADF materials in this paper for three-state TADF system (assuming $R_{D E}^{D F} \approx 1$ ).

\begin{tabular}{|c|c|c|c|c|c|c|c|c|c|c|}
\hline & & $\Phi_{P F}$ & $\Phi_{D F}$ & $\begin{array}{c}\boldsymbol{k}_{\boldsymbol{p}} \\
\left(10^{7} \mathrm{~s}^{-1}\right)\end{array}$ & $\begin{array}{c}\boldsymbol{k}_{\boldsymbol{d}} \\
\left(10^{5} \mathrm{~s}^{-1}\right)\end{array}$ & $\begin{array}{c}\boldsymbol{k}_{\boldsymbol{r}}^{\boldsymbol{S}} \\
\left(10^{7} \mathrm{~s}^{-1}\right)\end{array}$ & $\begin{array}{c}\boldsymbol{k}_{\boldsymbol{n} \boldsymbol{r}}^{\boldsymbol{S}} \\
\left(10^{6} \mathrm{~s}^{-1}\right)\end{array}$ & $\begin{array}{c}\boldsymbol{k}_{\boldsymbol{I S C}} \\
\left(10^{7} \mathrm{~s}^{-1}\right)\end{array}$ & $\begin{array}{c}\boldsymbol{k}_{n r}^{T} \\
\left(10^{4} \mathrm{~s}^{-1}\right)\end{array}$ & $\begin{array}{r}\boldsymbol{k}_{\boldsymbol{R} I S C} \\
\left(10^{5} \mathrm{~s}^{-1}\right)\end{array}$ \\
\hline \multirow{4}{*}{$\begin{array}{l}\text { 4CzIPN } \\
\quad \text { in toluene }\end{array}$} & original & $0.21^{\mathrm{a}}$ & 0.65 & \multirow{4}{*}{8.850} & \multirow{4}{*}{2.174} & 1.8 & - & 7.0 & - & 8.8 \\
\hline & SSA-1 & $0.21^{\mathrm{a}}$ & 065 & & & 1.858 & 0 & 6.991 & 3.853 & 8.517 \\
\hline & & & & & & 1.858 & 3.025 & 6.689 & 0 & 8.903 \\
\hline & exact & $0.21^{\mathrm{a}}$ & 0.65 & & & 1.858 & 3.190 & 6.61 & 0 & 8.836 \\
\hline \multirow{4}{*}{$\begin{array}{l}5 \mathrm{CzBN} \\
\quad \text { in toluene }\end{array}$} & original & $0.07^{\mathrm{a}}$ & 0.68 & \multirow{4}{*}{26.32} & \multirow{4}{*}{0.214} & 1.9 & - & 25 & - & 2.2 \\
\hline & & & & & & 1.842 & 0 & 24.47 & 0.574 & 2.232 \\
\hline & SDA-1 & 0.07 & 0.00 & & & 1.842 & 6.140 & 23.86 & 0 & 2.289 \\
\hline & exact & $0.07^{\mathrm{a}}$ & 0.68 & & & 1.842 & 6.160 & 23.84 & 0 & 2.288 \\
\hline \multirow{7}{*}{$\begin{array}{l}5 \mathrm{CzBN} \\
20 \mathrm{wt} \% \\
\text { in } \mathrm{mCBP}\end{array}$} & original & 0.16 & 0.65 & \multirow{7}{*}{31.25} & \multirow{7}{*}{0.980} & 5.0 & - & 26 & 4.1 & 3.6 \\
\hline & & & & & & 5.000 & 0 & 26.24 & 2.218 & 4.730 \\
\hline & SSA-1 & 0.16 & 0.65 & & & 5000 & 1173 & 2507 & 0 & 4052 \\
\hline & & & & & & 5.006 & 0 & 26.24 & 2.218 & 4.735 \\
\hline & SSA-2 & 0.160 & 0.650 & & & & & & & \\
\hline & & & & & & 5.006 & 11.74 & 25.07 & 0 & 4.957 \\
\hline & exact & 0.160 & 0.650 & & & 5.006 & 11.74 & 25.03 & 0 & 4.957 \\
\hline \multirow{8}{*}{$\begin{array}{l}3 \mathrm{Cz} 2 \mathrm{DPhCzBN} \\
20 \mathrm{wt} \% \\
\text { in } \mathrm{mCBP}\end{array}$} & original & 0.14 & 0.80 & \multirow{8}{*}{22.22} & \multirow{8}{*}{1.770} & 3.0 & - & 19 & 4.1 & 9.9 \\
\hline & & & & & & 1.552 & 0 & 19.11 & 1.235 & 11.76 \\
\hline & SSA-1 & 0.14 & 0.80 & & & & & & & \\
\hline & & & & & & 1.552 & 19.86 & 18.91 & 0 & 11.88 \\
\hline & & & & & & 3.125 & 0 & 19.10 & 1.236 & 11.717 \\
\hline & SSA-2 & 0.141 & 0.799 & & & & & & & \\
\hline & & & & & & 3.125 & 19.95 & 18.90 & 0 & 11.83 \\
\hline & exact & 0.141 & 0.799 & & & 3.125 & 19.95 & 18.80 & 0 & 11.83 \\
\hline \multirow{7}{*}{$\begin{array}{l}5 \mathrm{Cz}-\mathrm{TRZ} \\
\quad \text { in toluene }\end{array}$} & original & 0.031 & 0.889 & \multirow{7}{*}{24.39} & \multirow{7}{*}{2.439} & 0.544 & - & 17 & - & 150 \\
\hline & & & & & & 0.544 & 0 & 17.00 & 4.345 & 155.8 \\
\hline & SSA-1 & 0.031 & 0.889 & & & 0.544 & 0.473 & 16.95 & 0 & 156.2 \\
\hline & & & & & & 0.591 & 0 & 16.95 & 4.357 & 143.4 \\
\hline & SSA-2 & 0.034 & 0.886 & & & & & & & \\
\hline & & & & & & 0.591 & 0.514 & 16.90 & 0 & 143.8 \\
\hline & exact & 0.034 & 0.886 & & & 0.591 & 0.514 & 15.52 & 0 & 143.8 \\
\hline \multirow{8}{*}{$\begin{array}{l}\text { TQ } \\
10 \mathrm{wt} \% \\
\quad \text { in DPEPO }\end{array}$} & original & 0.459 & 0.095 & \multirow{8}{*}{8.8} & \multirow{8}{*}{111} & - & - & - & - & 0.035 \\
\hline & & & & & & 5.216 & 0 & 6.148 & 0.743 & 0.034 \\
\hline & SSA-1 & 0.459 & 0.095 & & & & & & & \\
\hline & & & & & & 5.216 & 41.99 & 1.949 & 0 & 0.109 \\
\hline & & & & & & 5.216 & 0 & 6.148 & 0.743 & 0.034 \\
\hline & SSA-2 & 0.459 & 0.095 & & & & & & & \\
\hline & & & & & & 5.216 & 41.99 & 1.948 & 0 & 0.109 \\
\hline & exact & 0.459 & 0.095 & & & 5.216 & 41.99 & 1.948 & 0 & 0.109 \\
\hline
\end{tabular}




\begin{tabular}{|c|c|c|c|c|c|c|c|c|c|c|}
\hline & & $\Phi_{P F}$ & $\Phi_{D F}$ & $\begin{array}{c}\boldsymbol{k}_{\boldsymbol{p}} \\
\left(10^{7} \mathrm{~s}^{-1}\right) \\
\end{array}$ & $\begin{array}{c}\boldsymbol{k}_{\boldsymbol{d}} \\
\left(10^{5} \mathrm{~s}^{-1}\right) \\
\end{array}$ & $\begin{array}{c}\boldsymbol{k}_{\boldsymbol{r}}^{\boldsymbol{S}} \\
\left(10^{7} \mathrm{~s}^{-1}\right) \\
\end{array}$ & $\begin{array}{c}\boldsymbol{k}_{\boldsymbol{n} \boldsymbol{r}}^{\boldsymbol{S}} \\
\left(10^{6} \mathrm{~s}^{-1}\right) \\
\end{array}$ & $\begin{array}{c}\boldsymbol{k}_{I S C} \\
\left(10^{7} \mathrm{~s}^{-1}\right) \\
\end{array}$ & $\begin{array}{c}\boldsymbol{k}_{n \boldsymbol{r}}^{\boldsymbol{T}} \\
\left(10^{4} \mathrm{~s}^{-1}\right) \\
\end{array}$ & $\begin{array}{r}\boldsymbol{k}_{\boldsymbol{R} I S C} \\
\left(10^{5} \mathrm{~s}^{-1}\right) \\
\end{array}$ \\
\hline \multirow{8}{*}{$\begin{array}{l}\text { v-DABNA } \\
\text { in toluene }\end{array}$} & original & 0.82 & 0.08 & \multirow{8}{*}{24.39} & \multirow{8}{*}{2.439} & 20 & 22 & 2.3 & 0 & 2.0 \\
\hline & & & & & & 20.00 & 0 & 4.390 & 13.55 & 1.322 \\
\hline & SSA-1 & 0.82 & 0.08 & & & & & & & \\
\hline & & & & & & 20.00 & 22.22 & 2.168 & 0 & 2.677 \\
\hline & & & & & & 20.00 & 0 & 4.388 & 13.56 & 1.321 \\
\hline & SSA-2 & 0.820 & 0.080 & & & & & & & \\
\hline & & & & & & 20.00 & 22.22 & 2.166 & 0 & 2.677 \\
\hline & exact & 0.820 & 0.080 & & & 20.00 & 22.22 & 2.163 & 0 & 2.677 \\
\hline \multirow{7}{*}{$\begin{array}{c}\text { TMCz-BO } \\
30 \mathrm{wt} \% \\
\text { in PPF }\end{array}$} & original & 0.66 & 0.32 & \multirow{7}{*}{2.632} & \multirow{7}{*}{13.33} & 1.7 & - & 0.9 & - & 19 \\
\hline & & & & & & 1.737 & 0 & 0.895 & 7.843 & 19.01 \\
\hline & SSA-1 & 0.66 & 0.32 & & & 1737 & 0354 & 0859 & 0 & 1980 \\
\hline & & & & & & & & & & \\
\hline & & 0676 & & & & 1.780 & 0 & 0.852 & 8.236 & 18.50 \\
\hline & & & & & & 1.780 & 0.363 & 0.816 & 0 & 19.32 \\
\hline & exact & 0.676 & 0.304 & & & 1.780 & 0.363 & 0.762 & 0 & 18.14 \\
\hline \multirow{8}{*}{$\begin{array}{l}\text { TpAT-tFFO } \\
\text { in mCBP }\end{array}$} & original & 0.02 & 0.82 & \multirow{8}{*}{6.536} & \multirow{8}{*}{2.262} & 0.11 & 0.20 & 5.3 & - & 120 \\
\hline & & & & & & 0.131 & 0 & 6.405 & 3.694 & 94.65 \\
\hline & SSA-1 & 0.02 & 0.82 & & & & & & & \\
\hline & & & & & & 0.131 & 0.249 & 6.380 & 0 & 95.02 \\
\hline & & & & & & 0.149 & 0 & 6.387 & 3.705 & 82.84 \\
\hline & SSA-2 & 0.023 & 0.817 & & & & & 6358 & & 19? \\
\hline & & & & & & & & & & \\
\hline & exact & 0.023 & 0.817 & & & 0.149 & 0.284 & 5.549 & 0 & 83.21 \\
\hline \multirow{8}{*}{$\begin{array}{r}\mathrm{Br}-3 \mathrm{PXZ}-\mathrm{XO} \\
\text { in toluene }\end{array}$} & original & 0.033 & 0.42 & \multirow{8}{*}{20.00} & \multirow{8}{*}{20.41} & 0.68 & - & 19 & 120 & 260 \\
\hline & & & & & & 0.660 & 0 & 19.34 & 115.4 & 268.6 \\
\hline & SSA-1 & 0.033 & 0.42 & & & & & & & \\
\hline & & & & & & 0.660 & 7.970 & 18.54 & 0 & 280.1 \\
\hline & & & & & & 0.746 & 0 & 19.25 & 116.0 & 236.4 \\
\hline & SSA-2 & 0.037 & 0.416 & & & & & & & \\
\hline & & & & & & 0.746 & 9.005 & 18.35 & 0 & 247.9 \\
\hline & exact & 0.037 & 0.416 & & & 0.746 & 9.005 & 16.08 & 0 & 247.9 \\
\hline \multirow{8}{*}{$\begin{array}{l}\text { DiKTa } \\
\quad \text { in toluene }\end{array}$} & original & 0.25 & 0.01 & \multirow{8}{*}{19.61} & \multirow{8}{*}{0.435} & 4.9 & 140 & 0.75 & - & 0.46 \\
\hline & & & & & & 4.902 & 0 & 14.71 & 4.290 & 0.023 \\
\hline & SSA-1 & 0.25 & 0.01 & & & & & & & \\
\hline & & & & & & 4.902 & 139.5 & 0.754 & - & 0.452 \\
\hline & & & & & & 4.902 & 0 & 14.71 & 4.290 & 0.023 \\
\hline & SSA-2 & 0.25 & 0.01 & & & & & & & \\
\hline & & & & & & 4.902 & 139.5 & 0.754 & - & 0.452 \\
\hline & exact & 0.25 & 0.01 & & & 4.902 & 139.5 & 0.754 & 0 & 0.452 \\
\hline \multirow{8}{*}{$\begin{array}{l}\text { MCz-TXT } \\
\quad \text { in mCBP }\end{array}$} & original & $0.012^{\mathrm{b}}$ & 0.908 & \multirow{8}{*}{107.3} & \multirow{8}{*}{13.33} & 1.3 & - & 94 & - & 1100 \\
\hline & & & & & & 1.287 & 0 & 106.0 & 10.80 & 1021.0 \\
\hline & SSA-1 & $0.012^{\mathrm{b}}$ & 0.908 & & & & & & & \\
\hline & & & & & & 1.287 & 1.119 & 105.9 & 0 & 1022.2 \\
\hline & & & & & & 1.287 & 0 & 105.8 & 10.81 & 933.2 \\
\hline & SSA-2 & $0.013^{b}$ & 0.907 & & & & & & & \\
\hline & & & & & & 1.408 & 1.224 & 105.7 & 0 & 934.3 \\
\hline & exact & $0.013^{\mathrm{b}}$ & 0.907 & & & 1.408 & 1.224 & 96.51 & 0 & 934.3 \\
\hline
\end{tabular}

a) using the PLQY before introduce the inert gas. 
Table 2. Summary of rate equations provided in this paper for three-state TADF system.

\begin{tabular}{|c|c|c|}
\hline Entry & Equations & Comments \\
\hline 1 & $\begin{array}{c}k_{r}^{S}=k_{p} \Phi_{P F} \\
k_{n r}^{S}=k_{p}\left(1-\Phi_{P F}-\Phi_{I S C}\right) \\
k_{I S C}=k_{p} \Phi_{I S C} \\
k_{r}^{T}=k_{d} \frac{\Phi_{D E}\left(1-R_{D E}^{D F}\right)}{\Phi_{I S C}} \\
k_{n r}^{T}=k_{d}-\left(1-\Phi_{I S C}\right) k_{R I S C}-k_{r}^{T} \\
k_{R I S C}=\frac{k_{d} \Phi_{D E} R_{D E}^{D F}}{\Phi_{I S C} \Phi_{P F}}\end{array}$ & $\begin{array}{l}\text { Assumption: } k_{r}^{S}+k_{n r}^{S}+k_{I S C} \gg k_{R I S C} \\
\text { to approximate } k_{p} \approx k^{S} \text { and apply steady state } \\
\text { approximation. } \\
\text { Requirement to obtain all rate constants } \\
k_{I S C} \text { estimation or assuming } k_{n r}^{S}=0 \text { or } k_{n r}^{T}=0 \\
R_{D E}^{D F} \text { estimation or assuming } R_{D E}^{D F}=1 \\
\qquad \Phi_{r}^{S}=\Phi_{P F}=\frac{k_{d}\left(A_{p}+A_{d}\right)}{A_{p} k_{d}+A_{d} k_{p}} \Phi_{P L Q Y} \\
\qquad \Phi_{D E}=\frac{A_{d}\left(k_{p}-k_{d}\right)}{A_{p} k_{d}+A_{d} k_{p}} \Phi_{P L Q Y}\end{array}$ \\
\hline 2 & $\begin{array}{c}k_{r}^{S}=k_{p} \Phi_{P F} \\
k_{I S C}^{\text {Ave. }}=\frac{k_{p}}{2} \cdot \frac{\left[\Phi_{P L Q Y}\left(1-\Phi_{P F}\right)+\Phi_{D F}\right] \pm\left[\Phi_{P L Q Y}\left(1-\Phi_{P F}\right)-\Phi_{D F}\right]}{\Phi_{P L Q Y}}=k_{p}\left(1-\Phi_{P F}-\frac{\Phi_{D F}}{\Phi_{P Q Y}}\right) \\
k_{R I S C}^{A v e}=\frac{k_{d}}{2} \cdot \frac{\left[\Phi_{P L Q Y}\left(1-\Phi_{P F}\right)+\Phi_{D F}\right] \pm\left[\Phi_{P L Q Y}\left(1-\Phi_{P F}\right)-\Phi_{D E}\right]}{\Phi_{P F}\left(1-\Phi_{P F}\right)}=k_{d}\left(1-\frac{\Phi_{D F}}{1-\Phi_{P F}}\right)\end{array}$ & $\begin{array}{l}\text { Based on method } 1 \\
\text { Assumption: } k_{r}^{S}+k_{n r}^{S}+k_{I S C} \gg k_{R I S C} \\
\text { to approximate } k_{p} \approx k^{S} \text { and apply steady state } \\
\text { approximation. } \\
\qquad \Phi_{r}^{S}=\Phi_{P F}=\frac{k_{d}\left(A_{p}+A_{d}\right)}{A_{p} k_{d}+A_{d} k_{p}} \Phi_{P L Q Y} \\
\qquad \Phi_{D E}=\frac{A_{d}\left(k_{p}-k_{d}\right)}{A_{p} k_{d}+A_{d} k_{p}} \Phi_{P L Q Y}\end{array}$ \\
\hline 3 & $\begin{array}{c}k_{r}^{S}=k_{p} \Phi_{P F} \\
k_{n r}^{S}=k^{S}-k_{r}^{S}-k_{I S C} \\
k_{I S C}=\frac{-b-\sqrt{b^{2}-4 a c}}{2 a} \\
k_{R I S C}=\frac{\left(k_{p}-k^{S}\right)\left(k^{S}-k_{d}\right)}{k_{I S C}} \\
k_{r}^{T}=\frac{k_{p} k_{d} \Phi_{D E}\left(1-R_{D E}^{D F}\right)}{k_{I S C}+k_{p}-k^{S}} \\
k_{n r}^{T}=k_{p}+k_{d}-k^{S}-k_{R I S C}-k_{r}^{T} \\
k^{S} \text { can be obtained as the solution of cubic equation } \\
k^{S^{3}}+d k^{S^{2}}+e k^{S}+f=0\end{array}$ & 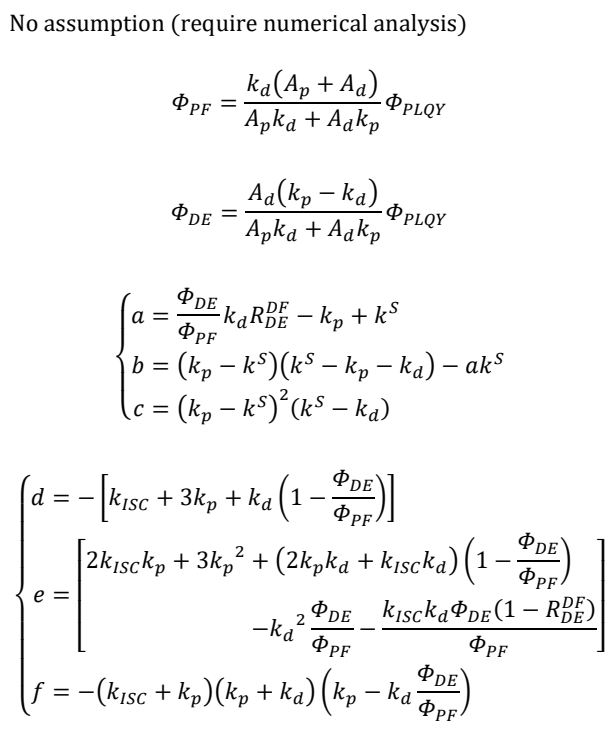 \\
\hline 4 & $\begin{array}{c}k_{r}^{S}=k_{p} \Phi_{P F} \\
k_{n r}^{S}=k_{p} \frac{\Phi_{P F}}{\Phi_{P L Q Y}}\left(1-\Phi_{P L Q Y}\right) \\
k_{I S C}=k_{p} \frac{\Phi_{D F}}{\Phi_{P L Q Y}}-k_{d} \frac{\Phi_{D F}}{\Phi_{P F}} \\
k_{R I S C}=k_{d} \frac{\Phi_{P L Q Y}}{\Phi_{P F}} \\
k_{n r}^{T}=0\end{array}$ & $\begin{array}{c}\Phi_{P F}=\frac{k_{d}\left(A_{p}+A_{d}\right)}{A_{p} k_{d}+A_{d} k_{p}} \Phi_{P L Q Y} \\
\Phi_{D E}=\frac{A_{d}\left(k_{p}-k_{d}\right)}{A_{p} k_{d}+A_{d} k_{p}} \Phi_{P L Q Y}\end{array}$ \\
\hline
\end{tabular}




\section{Supporting information}

\section{Exact Solution of Kinetic Analysis \\ for Thermally Activated Delayed Fluorescence Materials}

Youichi Tsuchiya, ${ }^{1 *}$ Stefan Diesing, ${ }^{2,3}$ Fatima Bencheikh, ${ }^{1}$ Yoshimasa Wada ${ }^{4}$ Paloma Dos Santos, ${ }^{2}$ Hironori Kaji, ${ }^{4}$ Eli Zysman-Colman, ${ }^{3}$ Ifor D. W. Samuel, ${ }^{2 *}$ and Chihaya Adachi ${ }^{1,5,6 *}$

\section{Contents:}

1. Summary of the methods to estimate $k_{R I S C}$ (Table S1)

2. Summary of the methods to estimate $\Phi_{I S C}$ (Table S2)

3. Conversion equation from quantum efficiency of prompt and delayed emission component commonly used to precise efficiency.

4. Relationship between efficiencies and overall efficiencies (for section 3)

5. Derivation of $\Phi_{I S C}^{n r T=0}$ for three-state analysis

6. Derivation of $k_{I S C}$ at the limit conditions $\left(k_{I S C}^{n r S=0}, k_{I S C}^{n r T=0}\right)$ for three-state analysis

7. Derivation of $k_{R I S C}$ at the limit conditions $\left(k_{R I S C}^{n r S=0}, k_{R I S C}^{n r T=0}\right)$ for three-state analysis

8. Definition of $k_{I S C}^{A v e}$ and $k_{R I S C}^{A v e}$

9. Detail calculation for $k_{I S C}^{A v e}$ and $k_{R I S C}^{A v e}$ for three-state analysis (approximating $\Phi_{P h o s} \approx 0$ for simplification)

10. Detail derivation of rate constants for four-state analysis among $S_{0}, S_{1}, T_{1}$ and $T_{n}$ 


\section{Summary of the methods to estimate $k_{R I S C}$ (Table S1)}

\begin{tabular}{|c|c|c|c|}
\hline Entry & Equations & Assumptions & Comments \\
\hline $1^{5}$ & $k_{R I S C}=\frac{1}{3} k_{I S C} \exp \left(\frac{-\Delta E_{S T}}{R T}\right)$ & $\begin{aligned} \cdot & \Phi_{I S C} \approx 1 \\
& \left(\Phi_{p} \ll \Phi_{d}, k_{n r}^{S} \approx 0\right) \\
\cdot & k_{I S C} \gg k_{r}^{S} \\
\cdot & k_{R I S C} \gg k_{r}^{T}+k_{n r}^{T}\end{aligned}$ & For metal complexes. \\
\hline $2^{6}$ & $k_{R I S C}=\frac{1}{3} k_{r}^{S} \exp \left(\frac{-\Delta E_{S T}}{R T}\right)$ & $\begin{aligned} \text { - } & \Phi_{P L Q Y} \approx 1 \\
& \left(k_{n r}^{S} \approx 0, k_{n r}^{T} \approx 0\right) \\
\cdot & \Phi_{p}<\Phi_{d},\left(k_{I S C} \gg k_{r}^{S}\right) \\
\cdot & \tau_{d}>1 \mu \mathrm{s} \\
\cdot & 0.05 \mathrm{eV}<\Delta E_{S T}<0.3 \mathrm{eV} \\
& \left(k_{r}^{T} \approx 0\right)\end{aligned}$ & \\
\hline $3^{7}$ & $k_{R I S C}=\frac{k_{p} k_{d}}{k_{I S C}} \cdot \frac{\Phi_{d}}{\Phi_{p}}=k_{d} \frac{\Phi_{d}}{\Phi_{p} \Phi_{I S C}}$ & $\begin{array}{l}\cdot k_{r}^{\mathrm{S}}, k_{n r}^{\mathrm{S}}, k_{I S C} \gg k_{R I S C}, k_{n r}^{T} \\
\cdot k_{r}^{\mathrm{T}} \approx 0\end{array}$ & \\
\hline 48 & $k_{R I S C}=\frac{k_{p} k_{d}}{k_{I S C}} \cdot \frac{\Phi_{d}}{\Phi_{p}}=k_{d} \frac{\Phi_{d}}{\Phi_{p} \Phi_{I S C}}$ & $\begin{array}{l}\cdot k_{r}^{\mathrm{S}}, k_{I S C} \gg k_{R I S C}, k_{n r}^{T} \\
\cdot k_{n r}^{\mathrm{S}} \approx 0 \\
\cdot k_{r}^{\mathrm{T}} \approx 0\end{array}$ & $\begin{array}{l}\text { Transient PL prompt must be } \\
\text { temperature independent. }\end{array}$ \\
\hline $5^{9}$ & $\begin{array}{l}\text { (1) } k_{R I S C}=\frac{k_{p} k_{d}}{k_{I S C}} \cdot \frac{\Phi_{d}}{\Phi_{p}} \\
\text { (2) } k_{R I S C}=\frac{k_{p} k_{d}}{k_{r}^{S}} \Phi_{P L Q Y}\end{array}$ & $\begin{array}{l}\cdot k_{p} \gg k_{d}, k_{r}^{\mathrm{T}} \approx 0 \\
\left(k_{r}^{\mathrm{S}}+k_{n r}^{\mathrm{S}}+k_{I S C} \gg k_{R I S C}+k_{n r}^{\mathrm{T}}\right) \\
\cdot k_{n r}^{\mathrm{S}} \approx 0, \Phi_{P L Q Y} \geq 0.9 \text { for }(1) \\
\cdot k_{n r}^{\mathrm{T}} \approx 0 \text { for }(2)\end{array}$ & $\begin{array}{l}k_{R I S C} \text { can be determined even } \\
\text { when } k_{r}^{\mathrm{S}}<k_{R I S C} \text {. }\end{array}$ \\
\hline $6^{10}$ & $k_{R I S C}=k_{d} \frac{1}{1-\Phi_{I S C}}=k_{d} \frac{\Phi_{p}+\Phi_{d}}{\Phi_{p}}$ & $\begin{array}{l}\cdot k_{\mathrm{n} r}^{\mathrm{T}} \approx 0 \\
\cdot k_{r}^{\mathrm{T}} \approx 0 \\
\text { - } k_{I S C} \gg k_{r}^{\mathrm{S}} \gg k_{n r}^{\mathrm{S}} \\
\text { - } k_{I S C} \gg k_{R I S C} \\
\text { - } \Phi_{d} / \Phi_{p} \geq 4\end{array}$ & \\
\hline 711 & $k_{R I S C}=\frac{k_{p}+k_{d}}{2}-\sqrt{\left(\frac{k_{p}+k_{d}}{2}\right)^{2}-k_{p} k_{d}\left(1+\frac{\Phi_{d}}{\Phi_{p}}\right)}$ & $\begin{array}{l}\cdot k_{\mathrm{n} r}^{\mathrm{T}} \approx 0 \\
\cdot k_{r}^{\mathrm{T}} \approx 0\end{array}$ & $\begin{array}{l}k_{R I S C} \text { can be determined even } \\
\text { when } k_{r}^{\mathrm{S}}<k_{R I S C} \text {. }\end{array}$ \\
\hline $8^{12}$ & $\begin{array}{c}\frac{\mathrm{d}\left[\mathrm{S}_{1}\right]}{\mathrm{d} t}=-\left(k_{r}^{\mathrm{S}}+k_{I S C}\right)\left[\mathrm{S}_{1}\right]+k_{R I S C}\left[\mathrm{~T}_{1}\right] \\
\frac{\mathrm{d}\left[\mathrm{T}_{1}\right]}{\mathrm{d} t}=k_{I S C}\left[\mathrm{~S}_{1}\right]-k_{R I S C}\left[\mathrm{~T}_{1}\right]\end{array}$ & $\begin{aligned} \cdot & \Phi_{P L Q Y} \approx 1 \\
& \left(k_{n r}^{S} \approx 0, k_{n r}^{T} \approx 0\right) \\
\cdot & k_{r}^{\mathrm{T}} \approx 0\end{aligned}$ & $\begin{array}{l}\text { Estimated by multi-parameter } \\
\text { fitting. }\end{array}$ \\
\hline 913 & $k_{R I S C}=\frac{k_{d 0}^{2}-k_{p 0} k_{d 0}}{k_{I S C}+k_{d 0}-k_{p 0}}$ & $\begin{array}{l}\cdot k_{n r}^{T} \approx 0 \\
\cdot k_{r}^{\mathrm{T}} \approx 0\end{array}$ & $\begin{array}{l}\text { Prepare the pristine and quencher } \\
\text { doped film. } \\
k_{p 0} \text { and } k_{d 0} \text { are prompt and } \\
\text { delayed decay rate of pristine film. } \\
k_{I S C} \text { is estimated by curve fitting } \\
\text { of delayed emission for quencher } \\
\text { doped film. } \\
\text { Other parameters such as exciton } \\
\text { diffusion coefficients and length } \\
\text { are also estimated. }\end{array}$ \\
\hline $10^{14}$ & $k_{R I S C}=k_{T} \Phi_{T A D F}$ & $\begin{array}{l}\text { - } \mathrm{S}_{1} \text { exciton is not quenched by } \\
\mathrm{O}_{2} \text {. }\end{array}$ & $\begin{array}{l}k_{T} \text { is triplet lifetime from } \\
\text { transient absorption spectra. } \\
\Phi_{T A D F} \text { is the difference of PLQY } \\
\text { presence and absence of } \mathrm{O}_{2} \text {. } \\
k_{R I S C} \text { is estimated as not an } \\
\text { elementary rate but an effective } \\
\text { rate, because ISC/RISC cycle is not } \\
\text { considered. }\end{array}$ \\
\hline
\end{tabular}

Reference numbers is corresponding to that in main text. 


\section{Summary of the methods to estimate $\Phi_{I S C}$ (Table S2)}

\begin{tabular}{|c|c|c|c|}
\hline Entry & Equations & Assumptions & Comments \\
\hline $1^{16}$ & $\Phi_{I S C}=\frac{\rho(\lambda)_{S S} k_{d}}{\varepsilon_{T}(\lambda) I_{a} l}$ & $\begin{array}{l}\text { - Depending on estimation } \\
\text { method of } \varepsilon_{T}(\lambda)^{17}\end{array}$ & \\
\hline $2^{18}$ & $\ln \left[\frac{I_{P F}}{I_{D F}}-\left(\frac{1}{\Phi_{I S C}}-1\right)\right]=\frac{\Delta E_{S T}}{R} \cdot \frac{1}{T}+\ln \left(\frac{k_{r}^{T}+k_{n r}^{T}}{\bar{k}_{R I S C}}\right)$ & $\begin{array}{l}\cdot k_{r}^{T}+k_{n r}^{T} \text { and } \Phi_{I S C} \text { are } \\
\text { temperature independent }\end{array}$ & $\begin{array}{l}\text { Temperature dependency of the } \\
\text { ratio of prompt and delayed } \\
\text { emission are measured to provide } \\
\text { the plot by using equation. } \\
\text { Then, you can find a best } \Phi_{I S C} \text { value } \\
\text { to obtain linearity of plot by } \\
\text { continuous variation of } \Phi_{I S C} \text {. }\end{array}$ \\
\hline $3^{19}$ & $\tau_{D F}=\tau_{P h o s}^{0}-\left(\frac{1}{\Phi_{I S C}}-1\right) \tau_{P h o s}^{0} \frac{I_{D F}}{I_{P F}}$ & $\begin{array}{l}\cdot k_{r}^{T}+k_{n r}^{T} \quad \text { and } \quad \Phi_{I S C} \text { are } \\
\text { temperature independent }\end{array}$ & $\begin{array}{l}\text { When } \tau_{P h o s}^{0} \text { is not provided, } \tau_{P h o s}^{0} \\
\text { and } \Phi_{I S C} \text { can be obtained by the } \\
\text { similar method of entry } 2 \text { with the } \\
\text { temperature dependency of } \tau_{D F} \\
\text { and } I_{D F} / I_{P F} \text {. }\end{array}$ \\
\hline 47 & $\begin{aligned} \Phi_{I S C}=\frac{3 N}{M-N} & \\
M & =\Phi_{E L}^{d} / \Phi_{E L}^{p} \\
N & =\Phi_{P L}^{d} / \Phi_{P L}^{p}\end{aligned}$ & & $\begin{array}{l}\text { Only thin film state can be applied. } \\
\text { The OLED device should be } \\
\text { fabricated using same emissive layer } \\
\text { with the thin layer for PL } \\
\text { measurement. }\end{array}$ \\
\hline $5^{20}$ & $\Phi_{I S C}=\frac{\Phi_{D F}+\Phi_{P h o s}}{\Phi_{r}^{S}+\Phi_{D F}+\Phi_{P h o s}}=\frac{\Phi_{D E}}{\Phi_{P L Q Y}}$ & - $k_{r}^{S}+k_{I S C} \gg k_{R I S C} \gg k_{r}^{T}+k_{n r}^{T}$ & $\begin{array}{l}\text { This method can be applicable to the } \\
\text { efficient TADF emitters. }\end{array}$ \\
\hline
\end{tabular}

Reference numbers is corresponding to that in main text. 
3. Conversion equation from quantum efficiency of prompt and delayed emission component commonly used to precise efficiency.

The quantum efficiency of prompt and delayed emission components, which have been used commonly in most of literature, were written as,

$$
\begin{gathered}
\Phi_{p}=\frac{A_{p} k_{d}}{A_{p} k_{d}+A_{d} k_{p}} \Phi_{P L Q Y}, \\
\Phi_{d}=\frac{A_{d} k_{p}}{A_{p} k_{d}+A_{d} k_{p}} \Phi_{P L Q Y} .
\end{gathered}
$$

From Eqs. 3.2, 3.3, S3.1 and S3.2 the conversion equations can be obtained as,

$$
\begin{gathered}
\Phi_{P F}=\Phi_{p}+\frac{k_{d}}{k_{p}} \Phi_{d}, \\
\Phi_{D E}=\left(1-\frac{k_{d}}{k_{p}}\right) \Phi_{d},
\end{gathered}
$$




\section{Relationship between efficiencies and overall efficiencies (for section 3)}

The efficiencies related to the distribution of triplet exciton can be described to Eqs. S4.1-S4.3.

$$
\begin{gathered}
\Phi_{R I S C}=\frac{k_{R I S C}}{k_{R I S C}+k_{r}^{T}+k_{n r}^{T}} . \\
\Phi_{r}^{T}=\frac{k_{r}^{T}}{k_{R I S C}+k_{r}^{T}+k_{n r}^{T}} . \\
\Phi_{n r}^{T}=\frac{k_{n r}^{T}}{k_{R I S C}+k_{r}^{T}+k_{n r}^{T}} .
\end{gathered}
$$

The overall efficiencies related to final distribution of triplet exciton can be described to Eqs. 4.17-4.19. From tease equations, the relationship between overall efficiencies and the efficiencies related to the distribution of $\mathrm{T}_{1}$ population as Eqs. S4.4-S4.6.

$$
\begin{gathered}
\Phi_{R I S C}{ }^{O E}=\frac{\left(1-\Phi_{I S C}\right) \Phi_{R I S C}}{1-\Phi_{I S C} \Phi_{R I S C}} . \\
\Phi_{r}^{T^{O E}}=\frac{\Phi_{r}^{T}}{1-\Phi_{I S C} \Phi_{R I S C}} . \\
\Phi_{n r}^{T}{ }^{O E}=\frac{\Phi_{n r}^{T}}{1-\Phi_{I S C} \Phi_{R I S C}} .
\end{gathered}
$$




\section{Derivation of $\Phi_{I S C}^{n r T=0}$ for three-state analysis}

From Eqs. 3.23, 3.24, and 3.25,

$$
\begin{aligned}
k_{n r}^{T}=0=k_{d}-(1 & \left.-\Phi_{I S C}^{n r T=0}\right) \frac{\left(\Phi_{D E}-\Phi_{P h o s}\right)}{\Phi_{P F}} \cdot \frac{k_{p} k_{d}}{k_{I S C}^{n r T=0}}-k_{d} \frac{\Phi_{P h o s}}{\Phi_{I S C}^{n r T=0}} \\
& =1-\frac{\Phi_{I S C}^{n r T=0} \Phi_{P h o s}-\Phi_{I S C}^{n r T=0} \Phi_{D E}+\Phi_{D E}-\Phi_{P h o s}-\Phi_{r}^{S} \Phi_{P h o s}}{\Phi_{P F} \Phi_{I S C}^{n r T=0}}
\end{aligned}
$$

therefore,

$$
\Phi_{I S C}^{n r T=0}=\frac{\Phi_{D E}-\Phi_{P h o s}\left(1+\Phi_{r}^{S}\right)}{\Phi_{P F}+\Phi_{D E}-\Phi_{P h o s}} .
$$


6. Derivation of $k_{I S C}$ at the limit conditions $\left(k_{I S C}^{n r S=0}, k_{I S C}^{n r T=0}\right)$ for three-state analysis From Eq. 3.10,

$$
\Phi_{I S C}^{n r S=0}=1-\Phi_{r}^{S}=1-\Phi_{P F}
$$

From Eqs. 3.22 and S6.1,

$$
k_{I S C}^{n r S=0}=k_{p}\left(1-\Phi_{P F}\right) .
$$

From Eqs. 3.22 and S5.1,

$$
k_{I S C}^{n r T=0}=k_{p} \frac{\Phi_{D E}-\Phi_{P h o s}\left(1+\Phi_{P F}\right)}{\Phi_{P F}+\Phi_{D E}-\Phi_{P h o s}} .
$$

When $\Phi_{\text {Phos }} \approx 0$ was employed,

$$
k_{I S C}^{n r T=0} \approx k_{p} \frac{\Phi_{D F}}{\Phi_{P F}+\Phi_{D E}}=k_{p} \frac{\Phi_{D F}}{\Phi_{P L Q Y}} .
$$

From Eqs. S6.2 and S6.4 the exchange equation of $k_{I S C}$ between the limit condition is provided as,

$$
k_{I S C}^{n r T=0}=k_{I S C}^{n r S=0} \frac{\Phi_{D F}}{\left(1-\Phi_{P F}\right) \Phi_{P L Q Y}} .
$$




\section{Derivation of $k_{R I S C}$ at the limit conditions $\left(k_{R I S C}^{n r S S=0}, k_{R I S C}^{n r T=0}\right)$ for three-state analysis}

From Eqs. 3.19 and 3.25,

$$
k_{R I S C}=k_{d} \frac{\Phi_{D E}-\Phi_{P h o s}}{\Phi_{P F} \Phi_{I S C}}
$$

From Eqs. S5.1, S6.1, and S7.1,

$$
\begin{gathered}
k_{R I S C}^{n r S=0}=k_{d} \frac{\Phi_{D E}-\Phi_{P h o s}}{\Phi_{P F}\left(1-\Phi_{P F}\right)} \\
k_{R I S C}^{n r T=0}=k_{d} \frac{\Phi_{D E}\left(\Phi_{P F}-\Phi_{D E}\right)-\Phi_{P h o s}\left(\Phi_{P F}-\Phi_{P h o s}\right)}{\Phi_{P F} \Phi_{D E}-\Phi_{P F} \Phi_{P h o s}\left(1+\Phi_{P F}\right)} .
\end{gathered}
$$

When $\Phi_{\text {Phos }} \approx 0$ was employed for simplification,

$$
\begin{gathered}
k_{R I S C}^{n r S=0}=k_{d} \frac{\Phi_{D F}}{\Phi_{P F}\left(1-\Phi_{P F}\right)}=k_{d} \frac{\Phi_{D F}}{\Phi_{P F} \Phi_{I S C}} \\
k_{R I S C}^{n r T=0}=k_{d} \frac{\Phi_{P F}+\Phi_{D F}}{\Phi_{P F}}=k_{d} \frac{\Phi_{P L Q Y}}{\Phi_{P F}} .
\end{gathered}
$$

Eqs. S7.4 and S7.5 are completely corresponding to the Goushi-Masui's equation and Dias' equation respectively (see Entry 3, 4, and 6 in Table S1). This means $k_{R I S C}$ values in the literatures estimated by their method with the different assumption of $\Phi_{n r}^{S}=0$ and $\Phi_{n r}^{T}=0$ can be compared by using Eq. S7.6. In this case, the efficiency values of $\Phi_{P F}$ and $\Phi_{D F}$ or $\Phi_{P L Q Y}$ with the ratio of $\Phi_{P F}$ and $\Phi_{D F}$ are requested to the literature.

$$
k_{R I S C}^{n r T=0}=k_{R I S C}^{n r S=0} \frac{\left(1-\Phi_{P F}\right) \Phi_{P L Q Y}}{\Phi_{D F}} .
$$


8. Definition of $\boldsymbol{k}_{I S C}^{A v e .}$ and $\boldsymbol{k}_{R I S C}^{A v e}$

$$
\begin{aligned}
& k_{I S C}^{A v g .} \equiv \frac{\left(k_{I S C}^{n r S=0}+k_{I S C}^{n r T=0}\right)}{2} \pm \frac{\left(k_{I S C}^{n r S=0}-k_{I S C}^{n r T=0}\right)}{2} . \\
& k_{R I S C}^{A v g .} \equiv \frac{\left(k_{R I S C}^{n r T=0}+k_{R I S C}^{n r S=0}\right)}{2} \pm \frac{\left(k_{R I S C}^{n r T=0}-k_{R I S C}^{n r S=0}\right)}{2} .
\end{aligned}
$$


9. Detail calculation for $k_{I S C}^{A v e}$ and $k_{R I S C}^{A v e .}$ for three-state analysis (approximating $\Phi_{P h o s} \approx 0$ for simplification)

From Eqs. S5.1, S6.4, and S8.1

$$
\begin{aligned}
k_{I S C}^{A v g .} & =\frac{1}{2}\left[k_{p}\left(1-\Phi_{P F}\right)+k_{p} \frac{\Phi_{D F}}{\Phi_{P L Q Y}}\right] \pm \frac{1}{2}\left[k_{p}\left(1-\Phi_{P F}\right)-k_{p} \frac{\Phi_{D F}}{\Phi_{P L Q Y}}\right] \\
& =\frac{k_{p}}{2} \cdot \frac{\left[\Phi_{P L Q Y}\left(1-\Phi_{P F}\right)+\Phi_{D F}\right] \pm\left[\Phi_{P L Q Y}\left(1-\Phi_{P F}\right)-\Phi_{D F}\right]}{\Phi_{P L Q Y}}
\end{aligned}
$$

From Eqs. 7.4, 7.5, and 8.2,

$$
\begin{aligned}
k_{R I S C}^{A v g .} & =\frac{1}{2}\left[k_{d} \frac{\Phi_{P L Q Y}}{\Phi_{P F}}+k_{d} \frac{\Phi_{D F}}{\Phi_{P F}\left(1-\Phi_{P F}\right)}\right] \pm \frac{1}{2}\left[k_{d} \frac{\Phi_{P L Q Y}}{\Phi_{P F}}-k_{d} \frac{\Phi_{D F}}{\Phi_{P F}\left(1-\Phi_{P F}\right)}\right] \\
& =\frac{k_{d}}{2} \cdot \frac{\left[\Phi_{P L Q Y}\left(1-\Phi_{P F}\right)+\Phi_{D F}\right] \pm\left[\Phi_{P L Q Y}\left(1-\Phi_{P F}\right)-\Phi_{D F}\right]}{\Phi_{r}^{S}\left(1-\Phi_{r}^{S}\right)} .
\end{aligned}
$$

Because the relationship of Eq. S6.5, $k_{I S C}^{\text {Ave. }}$ can be obtained from reported $k_{I S C}$ by using S9.1 as

$$
\begin{aligned}
& k_{I S C}^{A v g .}=k_{I S C}^{n r S=0} \frac{\left[\Phi_{P L Q Y}\left(1-\Phi_{P F}\right)+\Phi_{D F}\right] \pm\left[\Phi_{P L Q Y}\left(1-\Phi_{P F}\right)-\Phi_{D F}\right]}{2 \Phi_{P L Q Y}\left(1-\Phi_{r}^{S}\right)} . \\
& k_{I S C}^{A v g .}=k_{I S C}^{n r T=0} \frac{\left[\Phi_{P L Q Y}\left(1-\Phi_{P F}\right)+\Phi_{D F}\right] \pm\left[\Phi_{P L Q Y}\left(1-\Phi_{P F}\right)-\Phi_{D F}\right]}{2 \Phi_{D E}} .
\end{aligned}
$$

Because the relationship of Eq. S7.6, $k_{R I S C}^{A v e}$ can be obtained from reported $k_{R I S C}$ by using S9.2 as

$$
\begin{aligned}
& k_{R I S C}^{A v g .}=k_{R I S C}^{n r S=0} \frac{\Phi_{P L Q Y}\left(1-\Phi_{P F}\right)+\Phi_{D F} \pm \Phi_{P F}\left(1+\Phi_{P L Q Y}\right)}{2 \Phi_{D F}} . \\
& k_{R I S C}^{A v g .}=k_{R I S C}^{n r T=0} \frac{\Phi_{P L Q Y}\left(1-\Phi_{P F}\right)+\Phi_{D F} \pm \Phi_{P F}\left(1-\Phi_{P L Q Y}\right)}{2 \Phi_{P L Q Y}\left(1-\Phi_{P F}\right)} .
\end{aligned}
$$




\section{Detail derivation of rate constants for four-state analysis among $S_{0}, S_{1}, T_{1}$ and $T_{n}$}

Here, we derived the rate equations for the four-state analysis considering higher triplet state $\left(\mathrm{T}_{\mathrm{n}}\right)$ as an intermediate state of RISC state with the generally expected assumptions for the emitters.

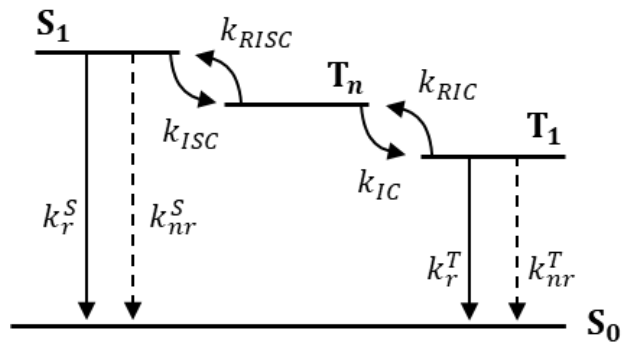

Figure $S 1$. Schematic diagram for four-state analysis of $S_{0}, S_{1}, T_{1}$ and $T_{n}$.

When we assume direct ISC/RISC process between $S_{1}$ and $T_{1}$ state and direct radiative/nonradiative process from $T_{n}$ to $S_{0}$ are forbidden, those decay pass can be eliminated. These assumptions should be related to the El-Saied's and Kasha's rules (Figure S1). The global decay rate from each excited state in the absence of exciton-formation processes can be formulated as

$$
\begin{gathered}
\frac{d\left[\mathrm{~S}_{1}\right]}{d t}=-k_{r}^{S}\left[\mathrm{~S}_{1}\right]-k_{n r}^{S}\left[\mathrm{~S}_{1}\right]-k_{I S C}\left[\mathrm{~S}_{1}\right]+k_{R I S C}\left[\mathrm{~T}_{\mathrm{n}}\right], \\
\frac{d\left[\mathrm{~T}_{\mathrm{n}}\right]}{d t}=-k_{R I S C}\left[\mathrm{~T}_{\mathrm{n}}\right]-k_{I C}\left[\mathrm{~T}_{\mathrm{n}}\right]+k_{I S C}\left[\mathrm{~S}_{1}\right]+k_{R I C}\left[\mathrm{~T}_{1}\right], \\
\frac{d\left[\mathrm{~T}_{1}\right]}{d t}=-k_{r}^{T}\left[\mathrm{~T}_{1}\right]-k_{n r}^{T}\left[\mathrm{~T}_{1}\right]-k_{R I C}\left[\mathrm{~T}_{1}\right]+k_{I C}\left[\mathrm{~T}_{\mathrm{n}}\right],
\end{gathered}
$$

where $\left[\mathrm{T}_{\mathrm{n}}\right]$ is the densities of $\mathrm{T}_{\mathrm{n}}$ excitons, $k_{I C}$ and $k_{R I C}$ are the rate constants for internal conversion (IC) and reverse-internal conversion (RIC) processes. In this system, all three components of $\left[\mathrm{S}_{1}\right],\left[\mathrm{T}_{\mathrm{n}}\right]$, and $\left[\mathrm{T}_{1}\right]$ should be provide as the tri-exponential curves. Therefore, the emission decay can be fit with a tri-exponential curve as,

$$
I(t)=A_{p} \exp \left(-k_{p} t\right)+A_{d 1} \exp \left(-k_{d 1} t\right)+A_{d 2} \exp \left(-k_{d 2} t\right),
$$

where $A_{p}, A_{d 1}, A_{d 2}, k_{p}, k_{d 1}$, and $k_{d 2}$ are the pre-exponential factors $(A)$ and decay rates $(k)$ for prompt $(p)$, primary delayed $(d 1)$, and secondary delayed $(d 2)$ components. To obtain the quantum efficiency of prompt $\left(\Phi_{P F}\right)$, primary delayed $\left(\Phi_{D E 1}\right)$, and secondary $\left(\Phi_{D E 2}\right)$ delayed components, Eq. S10.4 should be rewritten by,

$$
\begin{aligned}
I(t)=\left(A_{p}+A_{d 1}+A_{d 2}\right) & \exp \left(-k_{p} t\right) \\
+\left(A_{d 1}+A_{d 2}\right) & {\left[-\exp \left(-k_{p} t\right)+\exp \left(-k_{d 1} t\right)\right] } \\
& +A_{d 2}\left[-\exp \left(-k_{d 1} t\right)+\exp \left(-k_{d 2} t\right)\right] .
\end{aligned}
$$

In this form, the first, second, and third terms are exactly corresponding to the prompt, primary delayed and secondary delayed components, respectively, and each efficiency can be estimated as Eqs. S10.6-S10.8, 


$$
\begin{aligned}
& \Phi_{P F}= \frac{\frac{A_{p}+A_{d 1}+A_{d 2}}{k_{p}}}{\frac{A_{p}+A_{d 1}+A_{d 2}}{k_{p}}+\frac{A_{d 1}+A_{d 2}}{k_{d 1}}-\frac{A_{d 1}+A_{d 2}}{k_{p}}+\frac{A_{d 2}}{k_{d 2}}-\frac{A_{d 2}}{k_{d 1}}} \Phi_{P L Q Y} \\
&= \frac{\left(A_{p}+A_{d 1}+A_{d 2}\right) k_{d 1} k_{d 2}}{A_{p} k_{d 1} k_{d 2}+A_{d 1} k_{p} k_{d 1}+A_{d 2} k_{p} k_{d 2}} \Phi_{P L Q Y} . \\
& \frac{A_{d 1}+A_{d 2}}{k_{d 1}}-\frac{A_{d 1}+A_{d 2}}{k_{p}} \\
& \Phi_{D E 1}=\frac{\frac{A_{p}+A_{d 1}+A_{d 2}}{k_{p}}+\frac{A_{d 1}+A_{d 2}}{k_{d 1}}-\frac{A_{d 1}+A_{d 2}}{k_{p}}+\frac{A_{d 2}}{k_{d 2}}-\frac{A_{d 2}}{k_{d 1}}}{\left.A_{P L Q Y}+A_{d 2}\right)\left(k_{p}-k_{d 1}\right) k_{d 2}} \Phi_{P L Q Y} . \\
& \Phi_{D E 2}=\frac{\frac{A_{d 2}}{k_{d 2}}-\frac{A_{d 2}}{k_{d 1}}}{\frac{A_{p}+A_{d 1}+A_{d 2} k_{d 2}+A_{d 1} k_{p} k_{d 1}+A_{d 2} k_{p} k_{d 2}}{k_{p}}+\frac{A_{d 1}+A_{d 2}}{k_{d 1}}-\frac{A_{d 1}+A_{d 2}}{k_{p}}+\frac{A_{d 2}}{k_{d 2}}-\frac{A_{d 2}}{k_{d 1}}} \Phi_{P L Q Y} \\
&= \frac{A_{d 2}\left(k_{d 1}-k_{d 2}\right) k_{p}}{A_{p} k_{d 1} k_{d 2}+A_{d 1} k_{p} k_{d 1}+A_{d 2} k_{p} k_{d 2}} \Phi_{P L Q Y} .
\end{aligned}
$$

Under the assumption of $k_{r}^{S}+k_{n r}^{S}+k_{I S C} \gg k_{R I S C}$ with the restriction condition of $\left[\mathrm{S}_{1}\right] \gg\left[\mathrm{T}_{\mathrm{n}}\right]\left(t \ll 1 / k_{p}\right)$, Eq. S10.1 can be rewritten as Eq. S10.9. Therefore, the singlet decay rate $\left(k^{S}\right)$ can be approximate to the prompt decay rate $\left(k_{p}\right)$, and the function of time can be written as Eq. S10.10.

$$
\begin{gathered}
\frac{d\left[\mathrm{~S}_{1}\right]}{\left[\mathrm{S}_{1}\right]} \approx\left(-k_{r}^{S}-k_{n r}^{S}-k_{I S C}\right) d t \\
{\left[\mathrm{~S}_{1}\right] \approx A_{S} \exp \left[-\left(k_{r}^{S}+k_{n r}^{S}+k_{I S C}\right) t\right]=A_{S} \exp \left(-k^{S} t\right) \approx A_{S} \exp \left(-k_{p} t\right),}
\end{gathered}
$$

where $A_{S}$ is a pre-exponential factor.

Next, we focus on the exponential decay of $\left[\mathrm{T}_{\mathrm{n}}\right]$. Eq. S10.2 can be rewritten as Eq. S10.11 under the assumption of $k_{r}^{S}+k_{n r}^{S}+k_{I S C} \gg k_{R I S C}$ and $k_{R I S C}+k_{I C} \gg k_{R I C}$ with the restriction condition of $\left[\mathrm{T}_{1}\right] \ll\left[\mathrm{T}_{\mathrm{n}}\right]$ and $\left[\mathrm{S}_{1}\right] \ll$ [ $\left.\mathrm{T}_{\mathrm{n}}\right]\left(1 / k_{p} \ll t \ll 1 / k_{d 1}\right)$. By the assumption of $k_{R I S C}+k_{I C} \gg k_{R I C}$, the term related to the $\mathrm{T}_{1}$ can be vanished.

$$
\frac{d\left[\mathrm{~T}_{\mathrm{n}}\right]}{\left[\mathrm{T}_{\mathrm{n}}\right]}=\left(-k_{R I S C}-k_{I C}+k_{I S C} \frac{\left[\mathrm{S}_{1}\right]}{\left[\mathrm{T}_{\mathrm{n}}\right]}\right) d t
$$

The time dependent term of $\left[\mathrm{S}_{1}\right] /\left[\mathrm{T}_{\mathrm{n}}\right]$ can be obtained as non-time-dependent value by the steady state approximation (SSA) of Eq. S10.1, $d\left[\mathrm{~S}_{1}\right] / d t \approx 0$.

$$
\frac{\left[\mathrm{S}_{1}\right]}{\left[\mathrm{T}_{\mathrm{n}}\right]} \approx \frac{k_{R I S C}}{k_{r}^{S}+k_{n r}^{S}+k_{I S C}} .
$$

This require the assumption some of $k_{r}^{S}, k_{n r}^{S}$, or $k_{I S C}$ is much larger than $k_{R I S C}$. When $\mathrm{T}_{\mathrm{n}}$ level is lying below $\mathrm{S}_{1}$ level, $k_{I S C} \gg k_{R I S C}$ is always approved because the relationship of exo- and endothermic process. If $k_{I S C} \gg k_{R I S C}$ is satisfied as a consequence, it should not matter whether $T_{n}$ level is lying above $S_{1}$ level. By using Eq. $S 10.12$, the time dependence for $\left[T_{n}\right]$ is provided as Eq. S10.13.

$$
\begin{aligned}
{\left[\mathrm{T}_{\mathrm{n}}\right](t) } & \approx A_{T n} \exp \left\{-\left[\left(1-\frac{k_{I S C}}{k_{r}^{S}+k_{n r}^{S}+k_{I S C}}\right) k_{R I S C}+k_{I C}\right] t\right\} \\
& =A_{T n} \exp \left(-k^{T n} t\right) \approx A_{T n} \exp \left(-k_{d 1} t\right)
\end{aligned}
$$

where $A_{T n}$ is a pre-exponential factor, $k^{T n}$ is a decay rate of $\mathrm{T}_{\mathrm{n}}$ state. Therefore, $k_{d 1}$ can be approximate as, 


$$
k_{d 1} \approx\left(1-\frac{k_{I S C}}{k_{r}^{S}+k_{n r}^{S}+k_{I S C}}\right) k_{R I S C}+k_{I C}
$$

Further, we focus on the exponential decay of [ $\left.\mathrm{T}_{1}\right]$. Eq. S10.3 can be rewritten as Eq. S10.15 under the assumption of $k_{R I S C}+k_{I C} \gg k_{R I C}$ with the restriction condition of $\left[\mathrm{T}_{\mathrm{n}}\right] \ll\left[\mathrm{T}_{1}\right] t \gg 1 / k_{d 2}$

$$
\frac{d\left[\mathrm{~T}_{1}\right]}{\left[\mathrm{T}_{1}\right]}=\left(-k_{r}^{T}-k_{n r}^{T}-k_{R I C}+k_{I C} \frac{\left[\mathrm{T}_{\mathrm{n}}\right]}{\left[\mathrm{T}_{1}\right]}\right) d t
$$

The time dependent term of $\left[\mathrm{T}_{\mathrm{n}}\right] /\left[\mathrm{T}_{1}\right]$ can be obtained as non-time-dependent value by the steady state approximation (SSA) of Eqs. S10.1 and S10.2, $d\left[\mathrm{~S}_{1}\right] / d t \approx 0$ and $d\left[\mathrm{~T}_{\mathrm{n}}\right] / d t \approx 0$, respectively; this is necessary to assume $k_{I C} \gg$ $k_{R I C}$ but it always approved because of the relationship of exo- and endothermic process.

$$
\frac{\left[\mathrm{T}_{\mathrm{n}}\right]}{\left[\mathrm{T}_{1}\right]} \approx \frac{k_{R I C}}{\left(1-\frac{k_{I S C}}{k_{r}^{S}+k_{n r}^{S}+k_{I S C}}\right) k_{R I S C}+k_{I C}} .
$$

By the similar process, observed $k_{d 2}$ can be written as Eq. S10.17.

$$
k_{d 2} \approx k^{T 1}=\left[1-\frac{k_{I C}}{\left(1-\frac{k_{I S C}}{k_{r}^{S}+k_{n r}^{S}+k_{I S C}}\right) k_{R I S C}+k_{I C}}\right] k_{R I C}^{T}+k_{r}^{T}+k_{n r}^{T} .
$$

The total decay efficiency of singlet excitons generated by photo-excitation is the sum of $\Phi_{r}^{S}\left(\approx \Phi_{P F}\right), \Phi_{n r}^{S}$ and $\Phi_{I S C}$.

$$
\Phi_{r}^{S}+\Phi_{n r}^{S}+\Phi_{I S C}=1 .
$$

The decay efficiency of $T_{n}$ excitons resulted from an ISC process is the sum of $\Phi_{R I S C}$ and the internal conversion $\left(\Phi_{I C}^{T}\right)$. It should be noted again here, we assumed that the $\mathrm{T}_{\mathrm{n}}$ excitons do not decay directly to the $\mathrm{S}_{0}$ state but rather through the $\mathrm{T}_{1}$ state, related to Kasha's rule.

$$
\Phi_{R I S C}+\Phi_{I C}^{T}=1 .
$$

Because of $k_{r}^{S}+k_{n r}^{S}+k_{I S C} \gg k_{R I S C}$, the RISC process controls the decay of $\mathrm{T}_{\mathrm{n}}$ excitons via the $\mathrm{S}_{1}$ state and $\mathrm{S}_{1}$ excitons generated by the RISC process rapidly decayed to $S_{0}$ or $T_{n}$ states according to Eq. S10.18. Therefore, the multiple ISC/RISC cycle is present. In this case, the efficiencies in Eq. S10.19 should be modified by using overall efficiencies (OEs), which are the final distributed exciton ratio between $S_{1}$ and $T_{n}$ population via ISC/RISC cycles; those are $\Phi_{R I S C}{ }^{O E}>\Phi_{R I S C}$ and $\Phi_{I C}^{T}{ }^{O E}>\Phi_{I C}^{T}$. Observed decay rate is an apparent value of $\mathrm{T}_{\mathrm{n}}$ decays, OEs are employed to the analysis. Therefore, $\Phi_{I S C}$ can be divided into $\Phi_{R I S C}{ }^{O E}$ and $\Phi_{I C}^{T}{ }^{O E}$, and the total efficiency is given by,

$$
\Phi_{R I S C}{ }^{O E}+\Phi_{I C}^{T}{ }^{O E}=1 .
$$

Similarly, the RIC process controls the decay of $T_{1}$ excitons via the $T_{n}$ state and $T_{n}$ excitons generated by the RIC process rapidly decayed to $\mathrm{S}_{1}$ or $\mathrm{T}_{1}$ states according to Eq. $\mathrm{S} 10.20$ because of $k_{R I S C}+k_{I C} \gg k_{R I C}$. Therefore, the multiple IC/RIC cycle is present. The efficiencies related to $\mathrm{T}_{1}$ and its OEs are written by Eqs. S10.21 and S10.22; those are $\Phi_{r}^{T^{O E}}>\Phi_{r}^{T}, \Phi_{n r}^{T}{ }^{O E}>\Phi_{n r}^{T}$ and $\Phi_{R I C}{ }^{O E}<\Phi_{R I C}$. Observed decay rate is an apparent value of $\mathrm{T}_{1}$ decay, OEs are employed to the analysis. Therefore, $\Phi_{I S C} \Phi_{I C}{ }^{O E}$ can be divided into $\Phi_{r}^{T^{O E}}, \Phi_{n r}^{T}{ }^{O E}$ and $\Phi_{R I C}{ }^{O E}$.

$$
\Phi_{r}^{T}+\Phi_{n r}^{T}+\Phi_{R I C}=1 .
$$




$$
\Phi_{r}^{T}{ }^{O E}+\Phi_{n r}^{T}{ }^{O E}+\Phi_{R I C}{ }^{O E}=1
$$

The fraction of $\mathrm{T}_{\mathrm{n}}$ exciton decay event via $\mathrm{S}_{1}\left(\Phi_{I S C} \Phi_{R I S C}{ }^{O E}\right)$ can decay either radiatively $\left(\Phi_{r}^{S}\right)$ or non-radiatively $\left(\Phi_{n r}^{S}\right)$, because $\Phi_{R I S C} O E$ is the finally distributed exciton ratio after considering the ISC/RISC cycle. Therefore, the primary delayed fluorescence $\left(\Phi_{D F 1}\right.$, i.e., primary delayed emission $\left.\Phi_{D E 1}\right)$ can be formulated as a function of the radiative fraction to the total efficiency, $\Phi_{r}^{S}+\Phi_{n r}^{S}$.

$$
\Phi_{D F 1}=\Phi_{D E 1}=\Phi_{I S C} \Phi_{R I S C}{ }^{O E} \frac{\Phi_{r}^{S}}{\Phi_{r}^{S}+\Phi_{n r}^{S}}=\Phi_{I S C} \Phi_{R I S C}{ }^{O E} \frac{\Phi_{r}^{S}}{1-\Phi_{I S C}} .
$$

Similarly, the fraction of $\mathrm{T}_{1}$ exciton decay event via $\mathrm{S}_{1}\left(\Phi_{I S C} \Phi_{I C}{ }^{O E} \Phi_{R I C}{ }^{O E}\right)$ can decay either $\Phi_{r}^{S}$ or $\Phi_{n r}^{S}$, because $\Phi_{R I C} O E$ is the finally distributed exciton ratio after considering both IC/RIC and ISC/RISC cycles (see Eq. S10.17). Therefore, the secondary delayed fluorescence $\left(\Phi_{D F 2}\right)$ can be formulated as a function of $\Phi_{r}^{S}$ to the $\Phi_{r}^{S}+\Phi_{n r}^{S}$.

$$
\Phi_{D F 2}=\Phi_{D E 2} R_{D E 2}^{D F 2}=\Phi_{I S C} \Phi_{I C}{ }^{O E} \Phi_{R I C}{ }^{O E} \frac{\Phi_{r}^{S}}{\Phi_{r}^{S}+\Phi_{n r}^{S}}=\Phi_{I S C} \Phi_{I C}{ }^{O E} \Phi_{R I C}{ }^{O E} \frac{\Phi_{r}^{S}}{1-\Phi_{I S C}} .
$$

where $R_{D E 2}^{D F 2}$ is a ratio in of secondary delayed fluorescence in secondary delayed emission, $\Phi_{D F 2} / \Phi_{D E 2}$. In the fourstate analysis model, the observed lifetime of secondary delayed fluorescence and phosphorescence are exactly the same $\left(1 / k_{d 2}\right)$ since they occur from the same origin of the $\mathrm{T}_{1}$ state. Therefore, both $\Phi_{D F 2}$ and $\Phi_{P h o s}$ contribute to the secondary delayed emission $\left(\Phi_{D E 2}\right)$. The total PL quantum efficiency $\left(\Phi_{P L Q Y}\right)$ is the sum of $\Phi_{r}^{S}, \Phi_{D F 1}$, and $\Phi_{D E 2}$. Based on the above analysis, all of the efficiencies related to the TADF process were presented in Eqs. S10.25-S10.32.

$$
\begin{aligned}
& \Phi_{r}^{S}=\frac{k_{r}^{S}}{k_{r}^{S}+k_{n r}^{S}+k_{I S C}}=\frac{k_{r}^{S}}{k_{p}} \\
& \Phi_{n r}^{S}=1-\Phi_{r}^{S}-\Phi_{I S C}=\frac{k_{n r}^{S}}{k_{r}^{S}+k_{n r}^{S}+k_{I S C}}=\frac{k_{n r}^{S}}{k_{p}} . \\
& \Phi_{I S C}=\frac{k_{I S C}}{k_{r}^{S}+k_{n r}^{S}+k_{I S C}}=\frac{k_{I S C}}{k_{p}} . \\
& \Phi_{R I S C}{ }^{O E}=\frac{\Phi_{D E 1}\left(1-\Phi_{I S C}\right)}{\Phi_{r}^{S} \Phi_{I S C}}=\frac{\left(1-\Phi_{I S C}\right) k_{R I S C}}{\left(1-\Phi_{I S C}\right) k_{R I S C}+k_{I C}}=\frac{\left(1-\Phi_{I S C}\right) k_{R I S C}}{k_{d 1}} . \\
& \Phi_{I C}{ }^{O E}=\frac{k_{d 2}}{k_{R I C}^{T}} \cdot \frac{\Phi_{D F 2}}{\Phi_{D F 1}}=\frac{k_{I C}}{\left(1-\Phi_{I S C}\right) k_{R I S C}+k_{I C}}=1-\Phi_{R I S C}{ }^{O E} . \\
& \Phi_{R I C}{ }^{O E}=\frac{\Phi_{R I S C}{ }^{O E} k_{R I C}^{T}}{\Phi_{R I S C}{ }^{O E} k_{R I C}^{T}+k_{r}^{T}+k_{n r}^{T}}=\frac{\Phi_{R I S C}{ }^{O E} k_{R I C}^{T}}{k_{d 2}} . \\
& \Phi_{r}^{T}=\frac{\Phi_{P h o s}}{\Phi_{I S C} \Phi_{I C}^{O E}}=\frac{\Phi_{D E 2}\left(1-R_{D E 2}^{D F 2}\right)}{\Phi_{I S C} \Phi_{I C}{ }^{O E}}=\frac{k_{r}^{T}}{\Phi_{R I S C}{ }^{O E} k_{R I C}+k_{r}^{T}+k_{n r}^{T}}=\frac{k_{r}^{T}}{k_{d 2}} . \\
& \Phi_{n r}^{T^{O E}}=\frac{1-\Phi_{P L Q Y}}{\Phi_{I S C} \Phi_{I C}{ }^{O E}}-\Phi_{n r}^{S} \frac{1-\Phi_{I S C} \Phi_{I C}{ }^{O E}\left(1-\Phi_{R I C}{ }^{O E}\right)}{\Phi_{I S C} \Phi_{I C}{ }^{O E}\left(1-\Phi_{I S C}\right)}=\frac{k_{n r}^{T}}{\Phi_{R I S C}{ }^{O E} k_{R I C}+k_{r}^{T}+k_{n r}^{T}}=\frac{k_{n r}^{T}}{k_{d 2}} .
\end{aligned}
$$

The corresponding rate constants are described by Eqs. S10.33-S10.40.

$$
\begin{gathered}
k_{r}^{S}=k_{p} \Phi_{r}^{S} . \\
k_{n r}^{S}=k_{p} \Phi_{n r}^{S}=k_{p}\left(1-\Phi_{r}^{S}-\Phi_{I S C}\right) .
\end{gathered}
$$




$$
\begin{gathered}
k_{I S C}=k_{p} \Phi_{I S C} . \\
k_{R I S C}=k_{d 1} \frac{\Phi_{R I S C}{ }^{O E}}{\left(1-\Phi_{I S C}\right)}=\frac{\Phi_{D F 1}}{\Phi_{r}^{S}} \cdot \frac{k_{p} k_{d 1}}{k_{I S C}} . \\
k_{I C}^{T}=k_{d 1}-\left(1-\Phi_{I S C}\right) k_{R I S C} \cdot \\
k_{R I C}^{T}=k_{d 2} \frac{\Phi_{R I C}^{T}{ }^{O E}}{\Phi_{R I S C}{ }^{O E}}=k_{d 2} \frac{\left(1-\Phi_{I S C}\right) \Phi_{D E 2} R_{D E 2}^{D F 2}}{\Phi_{r}^{S} \Phi_{I S C} \Phi_{R I S C}{ }^{O E} \Phi_{I C}^{T}{ }^{O E}} . \\
k_{r}^{T}=k_{d 2} \Phi_{r}^{T^{O E}}=k_{d 2} \frac{\Phi_{D E 2}\left(1-R_{D E 2}^{D F 2}\right)}{\Phi_{I S C} \Phi_{I C}^{T}{ }^{O E}} . \\
k_{n r}^{T}=k_{d 2} \Phi_{n r}^{T}{ }^{O E}=k_{d 2} \frac{1-\Phi_{P L Q Y}}{\Phi_{I S C} \Phi_{I C}^{T}}-k_{d 2} \Phi_{n r}^{S} \frac{1+\Phi_{I S C} \Phi_{I C}^{T}{ }^{O E}\left(\Phi_{R I C}^{T}{ }^{O E}-1\right)}{\Phi_{I S C} \Phi_{I C}^{T}{ }^{O E}\left(1-\Phi_{I S C}\right)} .
\end{gathered}
$$

The value of $R_{D E 2}^{D F 2}$ can be estimated by fitting the secondary delayed emission spectrum with the prompt fluorescence and phosphorescence spectra to provide the contribution of the phosphorescence to the secondary delayed emission. For the data collected at high temperature, $R_{D E 2}^{D F 2}$ can be approximate as 1.

Because we obtained above rate equations by minimum assumptions. We can employ the constraint $\Phi_{n r}^{S}=0$ or $\Phi_{n r}^{T}=0$ as limit conditions to provide the $\Phi_{I S C}$ values of $\Phi_{I S C}^{n r S=0}$ and $\Phi_{I S C}^{n r T=0}$, respectively, as below.

$$
\begin{gathered}
\Phi_{I S C}^{n r S=0}=1-\Phi_{r}^{S} . \\
\Phi_{I S C}^{n r T=0}=\frac{\Phi_{P L Q Y}-\Phi_{r}^{S}-\left(1-\Phi_{r}^{S}\right) \Phi_{P h o s}}{\Phi_{P L Q Y}-\Phi_{P h o s}} \approx \frac{\Phi_{P L Q Y}-\Phi_{r}^{S}}{\Phi_{P L Q Y}}, \quad\left(\because R_{D E 2}^{D F 2} \approx 1\right) .
\end{gathered}
$$

We can also calculate the average $k_{I S C}, k_{R I S C}, k_{I C}^{T}$ and $k_{R I C}^{T}$ values with the range between the limit conditions $\left(\Phi_{n r}^{S}=0\right.$ or $\left.\Phi_{n r}^{T}=0\right)$, when it is difficult to estimate $\Phi_{I S C}$. By using $\Phi_{I S C}^{n r S=0}$ and $\Phi_{I S C}^{n r T=0}, \Phi_{R I S C}^{n r S=0}$ and $\Phi_{R I S C}^{n r T=0}$ are estimated from Eq. S10.36. The average rate constants for ISC and RISC can be obtained from Eqs. S8.1 and S8.2, respectively. The average rate constants for ISC and RISC can be obtained by Eqs. S10.43 and S10.44.

$$
\begin{aligned}
& k_{I C}^{A v g .} \equiv \frac{\left(k_{I C}^{n r S=0}+k_{I C}^{n r T=0}\right)}{2} \pm \frac{\left(k_{I C}^{n r S=0}-k_{I C}^{n r T=0}\right)}{2} . \\
& k_{R I C}^{A v g .} \equiv \frac{\left(k_{R I C}^{n r T=0}+k_{R I C}^{n r S=0}\right)}{2} \pm \frac{\left(k_{R I C}^{n r T=0}-k_{R I C}^{n r S=0}\right)}{2} .
\end{aligned}
$$

When $\Phi_{\text {Phos }}$ is approximated as 0 (i.e., $R_{D E 2}^{D F 2} \approx 1$ ) for the simplification, these average values can be estimated as followed.

$$
\begin{gathered}
k_{I S C}^{A v g .}=\frac{k_{p}\left[2 \Phi_{P L Q Y}-\Phi_{P F}\left(1+\Phi_{P L Q Y}\right) \pm \Phi_{P F}\left(1-\Phi_{P L Q Y}\right)\right]}{2 \Phi_{P L Q Y}} . \\
k_{R I S C}^{A v g .}=\frac{k_{d 1} \Phi_{D F 1}\left[2 \Phi_{P L Q Y}-\Phi_{P F}\left(1+\Phi_{P L Q Y}\right) \pm \Phi_{P F}\left(1-\Phi_{P L Q Y}\right)\right]}{2 \Phi_{r}^{S}\left(1-\Phi_{P F}\right)\left(\Phi_{P L Q Y}-\Phi_{P F}\right)} . \\
k_{I C}^{A v g .}=k_{d 1}-\frac{k_{d 1} \Phi_{D F 1}\left[1+\Phi_{P L Q Y}-2 \Phi_{P F} \pm\left(1-\Phi_{P L Q Y}\right)\right]}{2\left(1-\Phi_{P F}\right)\left(\Phi_{P L Q Y}-\Phi_{P F}\right)} . \\
k_{R I C}^{A v g .}=\frac{k_{d 2}\left[2 \Phi_{D F 2}\left(1-\Phi_{P F}\right)+\Phi_{D F 1}\left(1-\Phi_{P L Q Y}\right) \pm \Phi_{D F 1}\left(1-\Phi_{P L Q Y}\right)\right]}{2 \Phi_{D F 1}\left(1-\Phi_{r}^{S}-\Phi_{D F 1}\right)} .
\end{gathered}
$$

